\title{
Review
}

\section{Food Security Interventions among Refugees around the Globe: A Scoping Review}

\author{
Christine Nisbet $^{1}$, Kassandra E. Lestrat ${ }^{2}$ and Hassan Vatanparast ${ }^{2,3, *(\mathbb{D})}$ \\ 1 Office of the Vice President Research, University of Saskatchewan, Saskatoon, SK S7N 0W9, Canada; \\ christine.nisbet@usask.ca \\ 2 College of Pharmacy and Nutrition, University of Saskatchewan, Saskatoon, SK S7N 5E5, Canada; \\ ke1547@mail.usask.ca \\ 3 School of Public Health, University of Saskatchewan, Saskatoon, SK S7N 2Z4, Canada \\ * Correspondence: vatan.h@usask.ca; Tel.: +1-306-966-8866
}

check for updates

Citation: Nisbet, C.; Lestrat, K.E.; Vatanparast, H. Food Security Interventions among Refugees around the Globe: A Scoping Review. Nutrients 2022, 14, 522. https:// doi.org/10.3390/nu14030522

Academic Editors: Joanne E. Cecil and Samantha Caton

Received: 29 September 2021

Accepted: 20 January 2022

Published: 25 January 2022

Publisher's Note: MDPI stays neutral with regard to jurisdictional claims in published maps and institutional affiliations.

Copyright: (C) 2022 by the authors. Licensee MDPI, Basel, Switzerland. This article is an open access article distributed under the terms and conditions of the Creative Commons Attribution (CC BY) license (https:// creativecommons.org/licenses/by/ $4.0 /)$.

\begin{abstract}
There are 26 million refugees globally, with as many as $80 \%$ facing food insecurity irrespective of location. Food insecurity results in malnutrition beginning at an early age and disproportionately affects certain groups such as women. Food security is a complex issue and must consider gender, policies, social and cultural contexts that refugees face. Our aim is to assess what is known about food security interventions in refugees and identify existing gaps in knowledge. This scoping review followed the guidelines set out in the PRISMA Extension for Scoping Reviews. We included all articles that discussed food security interventions in refugees published between 2010 and 2020. A total of 57 articles were eligible for this study with most interventions providing cash, vouchers, or food transfers; urban agriculture, gardening, animal husbandry, or foraging; nutrition education; and infant and young child feeding. Urban agriculture and nutrition education were more prevalent in destination countries. While urban agriculture was a focus of the FAO and cash/voucher interventions were implemented by the WFP, the level of collaboration between UN agencies was unclear. Food security was directly measured in 39\% of studies, half of which used the UN's Food Consumption Score, and the remainder using a variety of methods. As substantiated in the literature, gender considerations are vital to the success of food security interventions, and although studies include this in the planning process, few see gender considerations through to implementation. Including host communities in food security interventions improves the refugee-host relationship. Collaboration should be encouraged among aid organizations. To assess intervention efficacy, food security should be measured with a consistent tool. With the number of refugees in the world continuing to rise, further efforts are required to transition from acute aid to sustainability through livelihood strategies.
\end{abstract}

Keywords: food security; food insecurity; refugees; intervention; displaced people; asylum seekers; scoping review

\section{Introduction}

There are 26 million refugees ("someone who is unable or unwilling to return to their country of origin owing to a well-founded fear of being persecuted for reasons of race, religion, nationality, membership of a particular social group, or political opinion" [1]) around the world (approximately 50\% are children) along with another 45.7 million internally displaced people ("[those who] have not crossed a border to find safety. Unlike refugees, they are on the run at home" [2]) and 4.2 million asylum seekers ("someone whose request for sanctuary has yet to be processed" [3]) [4]. The top source countries of refugees as of 2020 include Syria, Venezuela, Afghanistan, South Sudan, and Myanmar [4]. While some refugees reside in camps, the vast majority live in makeshift cities and host communities in neighbouring countries, where rising tensions have been reported [5]. Some refugees are 
provided the opportunity to resettle in countries such as Canada or those in Europe and others are repatriated [6]. The instability of many countries around the world due to war, religious and cultural persecution, and environmental disasters continues to increase the numbers of people fleeing their homes every day.

Recent conflicts around the globe are creating larger numbers of refugees for more prolonged periods of time. In desperation, refugees pay to board unsafe, overloaded water vessels. Many do not make it across with the number of dead and missing at its highest of more than 5000 in 2016 [7]. Families are forced to separate, people are met with long wait times trying to enter refugee camps in neighbouring countries, and some countries close their borders forcing refugees to search for asylum elsewhere. The protracted nature of crises such as that of Afghanistan and Syria strains host countries and aid agencies, stretching resources thin and impacting health care, food security, and livelihoods.

The United Nations Refugee Agency (UNHCR) reports that $80 \%$ of the world's displaced people are in locations suffering from acute food insecurity and malnutrition [8]. The COVID-19 pandemic continues to exacerbate the situation. Food security exists when all people at all times have access to safe and nutritious food appropriate for culture and lifestyle [9]. Food security must be examined across four pillars: physical availability of food, economic and physical access to food, food utilization, and stability over time [10]. The right to food and food security cannot exist without addressing the restrictive laws and policies refugees face in many countries such as those around employment and freedom of movement [11], yet food security remains a complex issue.

Particular consideration is needed for the most vulnerable, including women and girls; children; lesbian, gay, bisexual, transgender, queer and/or questioning, intersex, asexual, two spirit, and others (LGBTQIA2S+); the elderly; and persons with disabilities [12,13]. Women and children are particularly at risk of violence, sexual exploitation, and abuse as families are often separated during migration and refugees are forced to seek help from smugglers and others who take advantage of them, and refugee camps have high population densities with limited services. LGBTQIA2S+ populations are discriminated against, harassed, abused, and murdered, particularly in countries with anti-LGBTQIA2S+ legislation [14]. The elderly and persons with disabilities face barriers when it comes to accessing resources-such as water if collection points are far from their shelter-healthcare, and other services [15]. Gender roles are important in terms of household finances and food security. All over the world, women have a slightly higher prevalence of food insecurity compared to men [16]. Women are likely to prioritize food needs of spouses and children while compromising their own $[17,18]$. Culture is another important consideration because a lack of culturally available foods can destabilize cultural identity, affecting both physical and mental health [19]. Food insecurity results in a double burden of disease where malnutrition in childhood is followed by early establishment of chronic diseases later in life. The 2020 Global Nutrition Report indicates that 149 million children less than five years of age are stunted, 50 million are wasted, and 40 million are overweight [20]. Malnutrition is very common in refugee children [21]. Information is available on women and children; however, LGBTQIA2S+, the elderly, and those with disabilities are often overlooked in the design and implementation of humanitarian aid [14,15,22].

Some people live their whole lives in refugee camps with little hope of an autonomous future, yet they do what they can with their limited resources to survive. In camps, refugees are reliant upon aid, provided rations, vouchers, or cash for food. In makeshift cities, they can remain isolated from the host community where significant tensions exist $[5,23]$. Supplemental and therapeutic feeding centres are common for infant and young child feeding, target both refugees and host communities, and have proven to be successful in addressing malnutrition [24]. A vital strategy is to work with host countries to provide refugees with documentation to allow them the same rights as other citizens so they can access basic necessities like education, healthcare, and employment [25]. Such approaches improve self-reliance and mental health and provide training opportunities for refugees to 
build the gap between market demand and refugee skills, considering gender and other social and cultural contexts [26].

In resettlement countries, food security remains an issue. Migrants find cultural foods expensive, hard to obtain, and although people often have cooking skills, the unfamiliarity of new foods and how to prepare them pose challenges [19]. Refugees are provided aid for a short period of time, but many barriers such as language and lack of recognition of education from their home country makes it difficult to land jobs that pay well. For example, preliminary data indicates that $70 \%$ of Syrian refugees in Canada experience food insecurity [27].

Many countries around the world are welcoming refugees and donating money towards helping those in need. For example, United Nations Agencies and nongovernmental organization (NGO) partners pledged $\$ 5.5$ billion USD to assist Syrian refugees in 2020 [28]. With the numbers of refugees continuing to rise year after year, we need to review how we are helping these vulnerable people. Therefore, it is important to assess the types of food security interventions and identify the gaps in research to inform future programming to maximize efficiency of resources and help the largest number of people by the greatest extent possible.

\section{Objectives}

The objective of this scoping review is to assess what is known about food security interventions in refugees and identify existing gaps in knowledge.

Although our ultimate interest is refugees, interventions aimed towards other populations such as asylum seekers and displaced persons would be similar and so were also included. Interventions included formal interventions from research and humanitarian aid agencies such as cash and food transfers, food vouchers, urban agriculture, community gardens and kitchens. We are interested in knowing what interventions are most successful for refugees. We know that Community Based Participatory Research should be prioritized for successful interventions, placing the population of interest at the core, and engaging them throughout the entire research process. Therefore, we also included informal interventions implemented by refugees themselves such as the development of informal economies ("the diversified set of economic activities, enterprises, jobs, and workers that are not regulated or protected by the state...[including] wage employment in unprotected jobs" [29]). Although we are interested in which interventions are most successful, we also need to know what has been attempted with minimal to no success. Therefore, instead of only including successful interventions, we included all interventions. Refugee food security is a global issue, thus our review includes interventions from all countries, keeping in mind that different types of interventions will be observed according to where the country is along the migration process, from point of entry countries to transit countries, to final destination countries.

\section{Methods}

This scoping review followed the guidelines set out in the PRISMA Extension for Scoping Reviews (PRISMA-ScR): Checklist and Explanation (2018) article [30].

\subsection{Eligibility Criteria}

Inclusion criteria for this scoping review included any article that discussed a food security intervention in refugees. Articles were excluded if they were published prior to 2010, were not available in the English language, were not about food security interventions in refugees, or were exploratory studies, protocol or framework papers, conference abstracts, or review articles. For articles published by UN agencies, only those with an accompanying evaluation were included to incorporate a measure of effectiveness of interventions. 


\subsection{Information Sources and Selection}

The search was executed on 29 June 2020 in Ovid MEDLINE, Global Health, Public Health Databases, SCOPUS, and CABI Abstracts Global Health (from Web of Science). The search strategies were developed in consultation with the research team and a librarian experienced in scoping reviews. A sample search strategy from Ovid MEDLINE can be found in Supplementary Table S1: Sample search strategy. Search results were exported to EndNote X9 3.3 and duplicates removed [31]. Articles published from 2010 to 2020 were scanned in the Journal of Refugee Studies, the Journal of Immigration and Refugee Studies, and the Emergency Nutrition Network. The reference lists of all included studies were scanned for articles published from 2010 to 2020 that met the eligibility criteria. Grey literature was also included by scanning United Nations (UN) websites including the UNHCR, the Food and Agriculture Organization of the UN (FAO), the World Food Programme of the UN (WFP), and the World Health Organization. The titles and abstracts were scanned for eligibility criteria by authors CNN and KEL while any disagreements were discussed amongst all authors (CNN, KEL, and HAV) until consensus was reached.

\subsection{Data Charting Process and Data Items}

A form was developed in Microsoft Excel to extract all necessary details from the included articles: study location, study design (sample sizes at the household/family level vs. individual level and in the intervention vs. evaluation including pre and post), food security measurement tool, participants (age and gender), whether or not the intervention considers gender and any other at-risk groups, outcomes/important results, and limitations. Authors CNN and KEL charted the data and updated the form in an iterative process.

\subsection{Synthesis of Results}

Results are presented using a series of tables and figures to best depict the different results.

\section{Results}

\section{Selection and Characteristics of Sources of Evidence}

The removal of duplicates left a total of 4134 citations from electronic databases, journal scans, and reference list searches. Scanning titles and abstracts based on the eligibility criteria outlined above resulted in the exclusion of 4001 articles. We then went through 133 full text articles, whereby another 76 were excluded for not being about refugees or not distinguishing refugees from other population groups (e.g., immigrants), not including an intervention (cross-sectional, exploratory, simulation), or being a review, opinion, or policy. Therefore, a total of 57 articles were eligible for this study (Figure 1).

Table 1 is organized by the first author's last name and provides details on the characteristics of all included articles including aim, study design, and outcomes. We examined articles by location and found that 32\% targeted refugee camps and/or settlements, 19\% were outside camps, $26 \%$ were both inside and outside camps, one article did not specify, and $21 \%$ were in destination countries (Figure 2). We also found that $67 \%$ of interventions targeted refugees only, while 33\% targeted both refugees and host communities. Only 47\% of the articles indicated a consideration for gender when designing and implementing the interventions (i.e., programs were targeted specifically to women and/or women were prioritized by being provided e-transfers to manage household expenses or given roles to oversee food distribution). Few studies mentioned other at-risk populations such as children not covered by IYCF programs, the elderly and persons with disabilities. None of the studies mentioned LGBTQIA2S+. We found that $26 \%$ used a mixture of cash, vouchers, or food transfers for the intervention while another $11 \%$ were cash only interventions and $2 \%$ were voucher only. We also found that $28 \%$ of interventions were on urban agriculture, gardening, animal husbandry, or foraging; $12 \%$ on a combination of nutrition education type interventions; $12 \%$ on infant and young child feeding; $4 \%$ focused solely on schoolbased nutrition; $2 \%$ on community kitchens specifically; $2 \%$ on food safety and energy; and $2 \%$ on informal economy/trading (Figure 3). Table 2 is organized by location and provides 
details on the emerging themes from our results including location, target population, intervention type, consideration for gender, and food security measurement tool. Results indicated that $55 \%$ of interventions in nondestination countries were led by UN agencies of which $64 \%$ involved cash and/or vouchers; $20 \%$ used urban agriculture, gardening, and animal husbandry; and 16\% were on infant and young child feeding and pregnancy. For destination countries, one was in Canada, one in Germany, two in Australia, and eight in the USA (Figure 4). All seven of the nutrition education interventions took place in destination countries, representing $58 \%$ of the destination country interventions. The other interventions in destination countries involved urban agriculture (25\%), infant and young child feeding and pregnancy $(8 \%)$, and cash $(8 \%)$.
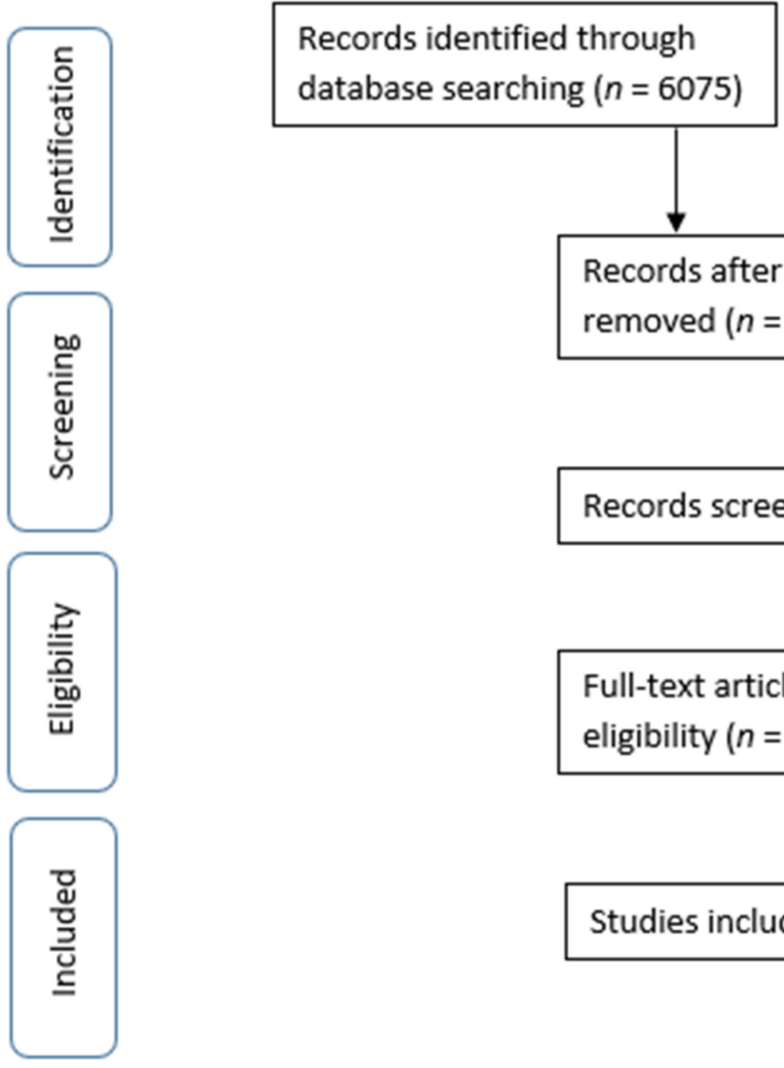

Records after duplicates

removed $(n=4134)$

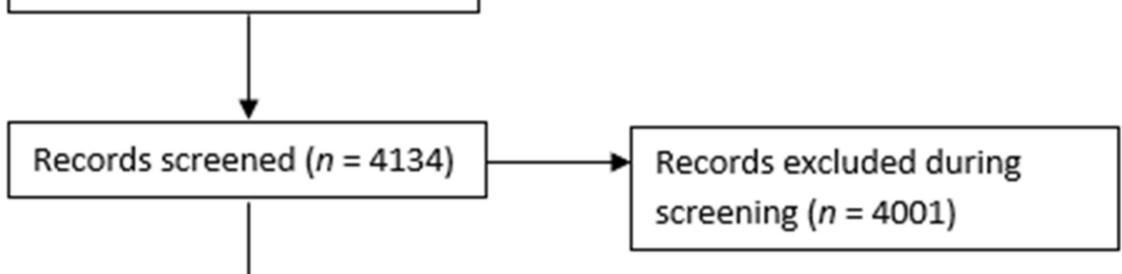

Full-text articles assessed for eligibility $(n=133)$ Full-text articles excluded,

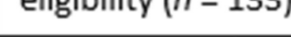
for eligibility reasons $(n=76)$

Figure 1. PRISMA Flow Diagram. 
Table 1. Characteristics of Sources of Evidence.

First Author, Year; Location Aim

Participants

Results

Positive perceptions of cash

Find effects of UN Refugee Agency (UNHCR) cash, UNICEF Child Cash Grant (CCG) on beneficiaries' lives: spending patterns, well-being. efficiency, effectiveness,

Abu Hamad B, 2017; Jordan Amman, Irbid, Mafraq and Zarqa [32] accountability of cash provided;

complementarity of and gaps in

programming for most vulnerable.
6 groups: 1. cash, CCG, and full-value vouchers; 2 . cash, CCG, and half-value vouchers; 3 . cash and full-value vouchers; 4 . cash and half-value vouchers; 5 . full-value vouchers only; 6 . half-value vouchers only. Eval-random selection, mixed methods: lit review, quantitative surveys, focus groups,

key informant interviews, case studies.

2114 household surveys: 627 cash, CCG, full-value vouchers; 418 cash CCG, half-value vouchers; 165 cash, full-value vouchers; 42 cash half-value vouchers; 251 full-value vouchers; 611 half-value vouchers. Purposive sampling for qualitative interviews-432 adults/children had different types of aid.

Alloush M, 2017; Rwanda, Kigeme, Nyabiheke, and Giheme camps [33]

Characterize demographics and income generating activities.
Cash camps: monthly transfers (m-VISA) on cell phones to gain cash, purchase goods/services. In-kind camps: monthly basket of maize, beans, oil, salt. Surveys: how camp economies interact with host-country economies; local economic impacts of in-kind vs. cash. Kigeme $=$ in-kind; Nyabiheke = cash; Giheme = cash
Random sample of households. Congolese refugees: 155-224 per each of 3 camps; host-country: 162-243 in economically relevant sectors $10 \mathrm{~km}$ of camps. Additional businesses: 15-23 refugees in each camp and $63-100$ hosts at main commercial sites within $10 \mathrm{~km}$ radii.

Promote caravans as safe for breastfeeding (bf)-privacy, support. Topics: nutrition for pregnant and lactating women, importance of bf, complementary feeding, feeding during illness
Pregnant women and mothers with children $<5$ years. Reached 15,600 mothers $>18$ month
Establish 3 caravans serving as mother-baby friendly space. ash $=$ avoid coping strategies (e.g

eat less, remove kids from school)

Borrowing money $\downarrow 79 \%$ to $26 \%$.

$90 \%$ said cash helped pay rent, $40 \%$

moved to better housing. $27 \%$ of all types of cash could not pay rent vs. $52 \%$ vouchers only. Cash, CCG les likely to have food shortages, forgo meat, eggs, dairy; more likely to have acceptable food security $(90 \%$ vs. $82 \%)$

$>80 \%$ of refugees sold food aid to purchase food, nonfood items. Refugees in cash camps better off than in-kind. Food security at Kigeme 14\%, Nyabiheke 39\%, Gihembe $60 \%$. Despite poor circumstances, economies form in camps-exchange of goods, services within/between camps and host economies.

Emphasized exclusive bf, time for complementary feeding. Identified bf difficulties, provided aid. $\uparrow$ awareness of risks of infant formula. Fortified food for 6-23 month distributed monthly; no 4th cycle-inadequate funds. 
Table 1. Cont.

\begin{tabular}{|c|c|c|c|c|}
\hline First Author, Year; Location & Aim & Intervention & Participants & Results \\
\hline $\begin{array}{l}\text { Aste N, 2017; Lebanon, } \\
\text { refugees in camps and } \\
\text { informal settlements [35] }\end{array}$ & $\begin{array}{l}\text { Improve food security (food } \\
\text { utilization) by testing energy } \\
\text { technologies, mainly related to } \\
\text { cooking, food preservation. }\end{array}$ & $\begin{array}{l}\text { Case A: Electricity previously few hrs/day } \\
\text { did not reach all households, unofficial } \\
\text { connections = unsafe exposure of electricity. } \\
\text { New system with security, safety. Case B: } \\
\text { previous lack of food preservation capacity, } \\
\text { illegal connection to grid, use of private } \\
\text { generators for lighting. Added system for } \\
\text { each family, charge controller, fuse for } \\
\text { system and user safety = electricity for } \\
\text { fridge, lights. }\end{array}$ & $\begin{array}{l}\text { Case A: Converted shopping mall } \\
\text { with } 134 \text { families ( } 670 \text { people). } \\
\text { Case B: } 82 \text { refugees in rural Lebanon } \\
\text { living in shelters and uncompleted } \\
\text { buildings. }\end{array}$ & $\begin{array}{c}\text { Case A: Diet diversity } \downarrow \text { due to } \downarrow \\
\text { value of vouchers. Diet diversity of } \\
\text { women }=\uparrow \text { trend-fridges stored } \\
\text { food longer, safely. Fridges } \downarrow \\
\text { expenses. Fridge internal temp not } \\
\text { as low as expected, preserve water, } \\
\text { bread. Case B: fridges preserve } \\
\text { some food for limited time due to } \downarrow \\
\text { food availability, unfamiliarity of } \\
\text { refrigeration. Food still perceived } \\
\text { safer, healthier. }\end{array}$ \\
\hline $\begin{array}{l}\text { Battistin F, 2018; Lebanon, } \\
\text { North, Beirut, Mt. Lebanon, } \\
\text { Bekaa, and South [36] }\end{array}$ & $\begin{array}{l}\text { Measure impact of Multipurpose } \\
\text { Cash Assistance Programme (MCA) } \\
\text { delivered by Lebanon Cash } \\
\text { Consortium at 6-month midline on } \\
\text { several proxies of physical and } \\
\text { material wellbeing: food security, } \\
\text { health, hygiene and housing. }\end{array}$ & $\begin{array}{l}\text { Quasi-experimental, Regression } \\
\text { Discontinuity Design; to compare outcomes } \\
\text { of households that received cash vs. those } \\
\text { who did not (non-MCA received vouchers). }\end{array}$ & $\begin{array}{l}20,000 \text { of } 25,000 \text { refugees eligible for } \\
\text { MCA were assisted due to lack of } \\
\text { funds. Eval: compared } 261 \text { MCA } \\
\text { and } 247 \text { non-MCA households; } \\
\text { most male-headed }(>75 \%) \text {. }\end{array}$ & $\begin{array}{l}\text { MCA } \uparrow \text { consumption of food, gas } \\
\text { for cooking. Food expenditures } 33 \% \\
\uparrow \text { for MCA vs. non-MCA. Food } \\
\text { security indicators not sig impacted } \\
\text { by MCA, but were for non-MCA. } \\
\text { No impact on food-related coping } \\
\text { strategies; both coped similarly. } \\
\text { MCA effective to address barriers } \\
\text { where markets functioning, flexible } \\
\text { to demand. }\end{array}$ \\
\hline $\begin{array}{l}\text { Betts A, 2020; Kenya, } \\
\text { Kalobeyei settlement and } \\
\text { Kakuma camp [37] }\end{array}$ & $\begin{array}{l}\text { Provide self-reliance to refugees, } \\
\text { greater refugee-host interaction } \\
\text { through development of Kalobeyei } \\
\text { settlement, planned for refugees } \\
\text { living on one side, hosts on the } \\
\text { other, with shared markets, schools, } \\
\text { hospitals in the middle. }\end{array}$ & $\begin{array}{l}\text { Bamba Chakula programme: monthly } \\
\text { mobile cash transfers for food at registered } \\
\text { shops. Kalobeyei: cash, corn-soya fortified } \\
\text { powder. Kakuma: cash, food basket. } \\
\text { Agriculture promotion programme } \\
\text { encouraged self-reliance, included kitchen } \\
\text { gardens, community plots. Eval: } \\
\text { quantitative survey to compare self-reliance } \\
\text { of recent arrivals, focus-groups, } \\
\text { semi-structured interviews. }\end{array}$ & $\begin{array}{l}2560 \text { surveys, } 15 \text { focus groups, } \\
>40 \text { semi-structured interviews with } \\
\text { refugees primarily from South } \\
\text { Sudan, smaller numbers from } \\
\text { Ethiopia Burundi, DR Congo, } \\
\text { Uganda, Sudan, Somalia; } \\
\text { nonrefugee stakeholders; gov } \\
\text { officials; host community. }\end{array}$ & $\begin{array}{l}\text { In Kalobeyei } 36 \% \text { of South Sudanese } \\
\text { had kitchen gardens vs. Kakuma } \\
24 \% \text {. Barriers: lack of water } 90 \% \text {, } \\
\text { seeds } 66 \% \text {, equipment } 29 \% \text {, soil } \\
\text { quality } 21 \% \text {. Rights to work } \\
\text { restricted in Kalobeyei. } 10 \% \text { earned } \\
\text { money, still low income. Public } \\
\text { services limited in both camps. } \\
\text { Acceptable diet diversity in } \\
\text { Kalobeyei } 66-76 \% \text {, Kakuma } 58 \% \text {. } \\
\text { Food insecurity in Kakuma } 93 \% \text {, } \\
\text { Kalobeyei } 78-90 \% \text {. }\end{array}$ \\
\hline
\end{tabular}


Table 1. Cont.

\begin{tabular}{|c|c|c|c|c|}
\hline First Author, Year; Location & Aim & Intervention & Participants & Results \\
\hline $\begin{array}{c}\text { Bloom JD, 2018; USA, North } \\
\text { Carolina [38] }\end{array}$ & $\begin{array}{l}\downarrow \text { social isolation, } \uparrow \text { access to } \\
\text { resources, adapt more generally to } \\
\text { USA food systems by facilitating } \\
\text { immigrant, refugee communities' } \\
\text { ability to apply healthy traditions in } \\
\text { a new context. }\end{array}$ & $\begin{array}{l}\text { Asset mapping workshop with women's } \\
\text { committee. Worked with } 2 \text { communities, } \\
\text { partner orgs } 1 \text { y to develop projects, } \\
\text { evaluate. Mosque home garden project: } \\
\text { attend class, provided materials, supplies. } \\
\text { Karen: connected with local nonprofit } \\
\text { incubator farm, provided training, } \\
\text { tools, seeds. }\end{array}$ & $\begin{array}{l}\text { Evaluative interviews with } \\
6 \text { women from women's committee } \\
\text { at local mosque, out of } 27 \text { total } \\
\text { participants. } 6 \text { interviews with } 7 \text { of } \\
8 \text { participants from } \\
\text { Karen community. }\end{array}$ & $\begin{array}{l}\text { Most mosque participants did not } \\
\text { produce enough veg to improve } \\
\text { intake. School garden food brought } \\
\text { to mosque, given out free. Karen } \\
\text { participants decreased store } \\
\text { purchases, improved access to } \\
\text { healthy, traditional food, shared } \\
\text { with 3-30 refugee families. }\end{array}$ \\
\hline
\end{tabular}


Table 1. Cont.

\section{First Author, Year; Location}

Dehnavi S, 2019; Lebanon, refugees and host communities [41]

\section{Alleviate hunger and}

underweight among participants by improving food security and economic resilience through improved food availability via home gardens.
Enhance the wellbeing of Persons of

Dunlop K, 2018; Greece, all [23]
Concern in Greece through access to protection-based and multi-sectorial humanitarian assistance.
Mixed methods. Eval outcomes: persons of concern can meet basic needs safely with dignity, choice; relationships with host communities improve. Eval examines negative coping strategies, links to local Greek economy through market cash injections via household surveys, focus groups, key informant interviews.
Recruitment: community liaison,

Combat physical and mental health conditions that accompany migration by developing a

Eggert LK 2015; USA, The Shenandoah Valley region of Virginia [42] snowballing. Coalition: fidelity in process,

satisfaction. Garden: fidelity to

construction, participation, satisfaction.

Seeds provided, gardeners contributed

tools, attended planting/training day.

Gardens assessed through season, community coalition to implement a community garden with apartment-dwelling refugees.
73 (72 female) households provided planting kits; $71 \%$ Syrian refugees, $29 \%$ Lebanese; $67 \%$ aged 30-45 years. 41 participants took part in the survey.

63,051 people received $€ 6.3 \mathrm{M}$

uantitative data examined 400 (44\% Syrian, 25\% Iraqi,

$16 \%$ Afghani, 9\% Iranian, $6 \%$ other household surveys. Qualitative data

from 6 focus groups, 21 key

informant interviews. 327 male,

73 female due to men more often listed as head of

household/card holder.

\section{Results}

Minorly alleviated underweight or hunger. Crop production,

cultivation low; $67 \% \uparrow$ availability of

fresh food, fruit/veg intake; diet

diversity. $29 \%$ satisfied: $61 \%$ lacked water, $56 \% \downarrow$ production than expected, 53\% limited inputs,

$17 \%$ unable to produce types of

plants wanted, $10 \%$ intending to sell products did not-low production.

Eval: most highly reported areas of spending: unmet needs at

baseline $=$ success of multipurpose cash grants. $71 \%$ felt cash partially met needs. Most frequently unmet needs: clothing $69 \%$, cigarettes $29 \%$ debt repayment $23 \%$. Most cash spent on food $(77 \%)$, $\uparrow$ with $\uparrow$ households, Syrians, Iraqis. Coping strategies: eat less

preferred/expensive food $70 \%, \downarrow$ meals/portions $45 \%$.

More veggies consumed, most donated some of their garden, some liked not having to go to the store,
5 gardeners began the season, 4 remained (1 moved) advice available. all wanted a larger plot of land to

garden. Two refugee residents observing community garden plots expressed interest in larger-scale urban farming. 
Table 1. Cont.

\begin{tabular}{|c|c|c|c|c|}
\hline First Author, Year; Location & Aim & Intervention & Participants & Results \\
\hline $\begin{array}{c}\text { El Harake MD, 2018; } \\
\text { Lebanon, cities of Majdal } \\
\text { Anjar, Saadneyil, and Bar } \\
\text { Elias in the Bekaa valley [43] }\end{array}$ & $\begin{array}{l}\text { Evaluate a 6-month pilot } \\
\text { school-based nutrition intervention } \\
\text { on changes in diet knowledge, } \\
\text { attitudes, behaviours of Syrian } \\
\text { refugee children in informal } \\
\text { primary schools in rural Lebanon; } \\
\text { explore the effect of the intervention } \\
\text { on diet intake, nutrition status } \\
\text { of children. }\end{array}$ & $\begin{array}{l}\text { Quasi-experimental design. } 2 \text { intervention } \\
\text { schools: health and nutrition education } \\
\text { bi-weekly, nutritious snacks. Control } \\
\text { school: usual curriculum, standard snack. } \\
\text { Interviews with children, mothers: } \\
\text { household sociodemographics, diet } \\
\text { knowledge, attitude, child behaviour, } \\
\text { anthropometric measures, diet intake. }\end{array}$ & $\begin{array}{c}\text { Data collected at baseline from } \\
296 \text { Syrian refugee students } \\
6-14 \text { years (grades } 4-6 \text { ). Data at } \\
\text { baseline and follow up available for } \\
203 \text { children. Sample size reduced to } \\
183 \text { due to clustering. At baseline, } \\
\text { mean age of children was } 11 \text { years, } \\
51 \% \text { female. }\end{array}$ & $\begin{array}{c}\text { Baseline: } 79 \% \text { severely food } \\
\text { insecure, } 3 \% \text { food secure. Greater } \\
\text { change in knowledge, body mass } \\
\text { index-for-age (z score) and } \\
\text { height-for-age (z scores) in } \\
\text { intervention vs. Control. Compared } \\
\text { to control, intervention children had } \\
\text { on average sig } \uparrow \text { mean changes in } \\
\text { daily intakes: kcal, dietary fiber, } \\
\text { protein, saturated fat, vit K, zinc, } \\
\text { calcium, magnesium. }\end{array}$ \\
\hline $\begin{array}{l}\text { Fander G, 2014; Jordan, } \\
6 \text { northern governates } \\
\text { (Amman, Zarqa, } \\
\text { Mafraq-including Za'atari } \\
\text { refugee camp, Irbid, Jerash } \\
\text { and Ajloun) [44] }\end{array}$ & $\begin{array}{l}\text { Protect children }<5 \text { years and } \\
\text { pregnant and lactating women } \\
\text { (PLW) by screening for malnutrition } \\
\text { and educating caregivers about } \\
\text { infant and young children } \\
\text { feeding practices. }\end{array}$ & $\begin{array}{l}\text { Pre-intervention assessment: breastfeeding } \\
\text { misconceptions. Project: education on } \\
\text { exclusive breastfeeding, correct/timely } \\
\text { introduction of complementary foods via } \\
\text { clinics with nutrition officer or similar; } \\
\text { support for mothers willing to re-lactate; } \\
\text { Super Cereal Plus supplement to treat } \\
\text { moderate acute malnutrition in kids } \\
<5 \text { years, PLW. }\end{array}$ & $\begin{array}{l}\text { Over } 10 \text { months, } \\
4690 \text { pregnant/lactating women } \\
\text { received education and } 919 \text { mothers } \\
\text { engaged in } \\
\text { breastfeeding counselling. }\end{array}$ & $\begin{array}{l}\text { Increase in breastfeeding } \\
\text { knowledge, but not in breastfeeding } \\
\text { practice. Out of } 46,383 \text { children } \\
\text { screened, } 69 \text { had severe acute } \\
\text { malnutrition, } 124 \text { had moderate } \\
\text { acute malnutrition. Out of } \\
\text { 10,088 PLW screened, } 457 \text { were } \\
\text { acutely malnourished. }\end{array}$ \\
\hline
\end{tabular}

Food and Agriculture Organization of the United

Nations, 2016; Lebanon,

Akkar, Tripoli, and the Bekaa regions [45]
Promote diversified and quality

food for vulnerable landless

households through micro-gardens.
6 micro-garden structures tested, as well as one method with no structure (plastic crate distributed for use as planter boxes). Initial training: technical support, follow-up of weekly site visits. Successes and/or failures of each method recorded, analyzed.
170 direct beneficiaries (76 vulnerable Lebanese and 94 displaced Syrians).
Vertical planting had lowest success, simplest structures best. Plastic crates cheapest, easiest, most successful, more easily accepted. Other factors impacted success: space, pest-resistant seed, reliable water supply, extreme weather protection. Micro-gardens $\uparrow$ quality of life, not a replacement for agriculture. Learnings: restrict to cooler seasons, use more pest-resistant leafy veg, herbs. 
Table 1. Cont.

\begin{tabular}{|c|c|c|c|c|}
\hline First Author, Year; Location & Aim & Intervention & Participants & Results \\
\hline $\begin{array}{l}\text { Food and Agriculture } \\
\text { Organization of the United } \\
\text { Nations, 2018; Uganda, } \\
\text { refugee settlements in the } \\
\text { northern and mid-western } \\
\text { regions [46] }\end{array}$ & $\begin{array}{l}\text { Improve food, nutrition, income } \\
\text { security of refugees, } \\
\text { host communities. }\end{array}$ & $\begin{array}{l}\text { Planting materials and inputs for } \\
\text { small-scale veg, staple food, poultry } \\
\text { production, preservation techniques } \\
\text { provided with construction, use of energy } \\
\text { saving stoves, training in entrepreneurship } \\
\text { and animal husbandry. }\end{array}$ & $\begin{array}{l}8000 \text { households of most vulnerable } \\
\text { refugee, host } \\
\text { community households. }\end{array}$ & $\begin{array}{c}\text { More diversified income sources; } \\
\uparrow \text { food security, diets; stronger } \\
\text { livelihoods of vulnerable } \\
\text { refugee/host communities. } \\
\text { Beneficiaries learned skills, } \\
\uparrow \text { knowledge, ameliorated } \\
\text { conservation practices allowed } \\
\text { women to stay closer to home } \downarrow \\
\text { gender-based violence. Improved } \\
\text { refugee-host relationships, market } \\
\text { access, economy. }\end{array}$ \\
\hline $\begin{array}{c}\text { Food and Agriculture } \\
\text { Organization of the United } \\
\text { Nations, 2020; DR Congo, } \\
\text { the provinces of Ituri and } \\
\text { Haut-Uélé [47] }\end{array}$ & $\begin{array}{l}\text { Empower South Sudanese refugees } \\
\text { through income-generating and } \\
\text { agricultural activities }\end{array}$ & $\begin{array}{l}\text { Participants provided tools, seeds; training } \\
\text { on agricultural, nutrition, healthy living } \\
\text { practices. Project used cash transfers to } \\
\text { facilitate access to goods, } \\
\text { improve livelihoods. }\end{array}$ & $\begin{array}{l}2000 \text { South Sudanese refugee } \\
\text { households, } 1000 \text { host households; } \\
\sim 15,000 \text { people. } 545 \text { refugee } \\
\text { households also provided goats to } \uparrow \\
\text { access to animal protein. }\end{array}$ & $\begin{array}{l}\text { Providing cash to rural peoples, } \\
\text { refugees allows them to meet needs } \\
\text { while waiting for harvests, diversify } \\
\text { livelihoods, invest in school for } \\
\text { children, healthcare, and financing } \\
\text { for small business ventures. }\end{array}$ \\
\hline $\begin{array}{l}\text { Ghattas } H, 2019 \text {; Lebanon, } \\
\text { refugee camps [48] }\end{array}$ & $\begin{array}{l}\text { Establish community kitchens (CKs) } \\
\text { as social enterprises-improve } \\
\text { mental health, income, food security, } \\
\text { women's empowerment; link with } \\
\text { school nutrition to improve kids' } \\
\text { diets, school } \\
\text { attendance, performance. }\end{array}$ & $\begin{array}{l}\text { Quasi-experimental, convenience sampling. } \\
2 \text { CKs with 1-wk training: hands-on food } \\
\text { safety, hygiene, nutrition education, } \\
\text { entrepreneurship. Intervention schools: } \\
\text { subsidized healthy food sold at school, } \\
\text { nutrition education. Control schools: } \\
\text { nutrition education. Evals with teachers, } \\
\text { parents, children. }\end{array}$ & $\begin{array}{l}\text { Community kitchen: } 51 \text { women } \\
\text { recruited, } 33 \text { completed the study. } \\
\text { School program: of } 847 \text { children } \\
\text { 5-15 years attending intervention } \\
\text { schools, } 714 \text { participated over } \\
2 \text { years. }\end{array}$ & $\begin{array}{l}\text { Participatory approach = compatible } \\
\text { work, home schedules for women. } \\
90 \% \text { of intervention and } 95 \% \text { of } \\
\text { control school parents responded } \\
\text { positively. Education sessions well } \\
\text { attended by children, not parents. } \\
\text { Children enjoyed snacks. Food } \\
\text { security results not presented. }\end{array}$ \\
\hline $\begin{array}{l}\text { Gichunge C, 2014; Australia, } \\
\text { East Queensland [49] }\end{array}$ & $\begin{array}{l}\text { Examine gardening as part of the } \\
\text { food environment of } \\
\text { African refugees. }\end{array}$ & $\begin{array}{l}\text { Qualitative study using in-depth interviews } \\
\text { and a questionnaire on socio-demographics. } \\
\text { Resettled African refugees who engaged in } \\
\text { home and community gardening and spoke } \\
\text { English or Swahili were recruited using } \\
\text { purposive sampling. }\end{array}$ & $\begin{array}{c}13 \text { gardeners ( } 85 \% \text { female) were } \\
\text { interviewed. } 3 \text { from South Sudan, } 1 \\
\text { from the DR Congo, and } 9 \\
\text { from Burundi. }\end{array}$ & $\begin{array}{l}3 \text { themes: food provision-gardens } \uparrow \\
\text { access to fresh/traditional food, } \\
\text { saved money; health } \\
\text { improvement-gardens helped } \\
\text { people stay active, relieve stress, } \uparrow \\
\text { self-efficacy; food environment } \\
\text { barriers-cost, small plots, } \\
\text { knowledge of new climate. }\end{array}$ \\
\hline
\end{tabular}


Table 1. Cont.

\begin{tabular}{|c|c|c|c|c|}
\hline First Author, Year; Location & Aim & Intervention & Participants & Results \\
\hline $\begin{array}{c}\text { Giordano, 2017; Jordan, } \\
\text { Amman, Irbid, Marfraq, and } \\
\text { Zarqa [50] }\end{array}$ & $\begin{array}{l}\text { Review model chosen to deliver } \\
\text { cash, identify themes of change for } \\
\text { recipients. Common Cash Facility } \\
\text { (CCF): platform for delivering cash, } \\
\text { provides orgs direct, equal access to } \\
\text { common financial service provider, } \\
\text { payment facility. }\end{array}$ & $\begin{array}{l}\text { By 2016, CCF delivered }>90 \% \text { of cash to } \\
\text { refugees outside camps in Jordan. Eval: } \\
\text { efficiency, effectiveness, relevance, } \\
\text { coverage, accountability, innovation using } \\
\text { data from post-distribution monitoring } \\
\text { surveys on usage patterns, effectiveness of } \\
\text { cash, recipient satisfaction. }\end{array}$ & Unclear. & $\begin{array}{l}\text { Compared to nonrecipients, } \\
\text { recipients: ate } \uparrow \text { meals } / \text { day, fruit, } \\
\text { eggs, meat; more diverse diets; } \downarrow \\
\text { coping strategies; } \uparrow \text { income, } \uparrow \text { assets, } \\
\uparrow \text { expenditures. } 62-73 \% \text { of } \\
\text { households ate } 2 \text { meals the previous } \\
\text { day, } 5-10 \% \text { ate } 1 .>50 \% \text { reported the } \\
\text { most important effect of cash } \\
\text { assistance was eating better. }\end{array}$ \\
\hline $\begin{array}{l}\text { Goh J, 2017; Germany, } \\
\text { Munich [51] }\end{array}$ & $\begin{array}{l}\text { Use unconditional cash transfers to } \\
\uparrow \text { knowledge of refugee spending } \\
\text { patterns to help aid orgs create more } \\
\text { effective programs. }\end{array}$ & $\begin{array}{l}\text { Distributed } € 60 \text { to each social welfare } \\
\text { participant to spend without limitations } \\
\text { over ten days. Participants were divided } \\
\text { into } 3 \text { monthly income levels: }<€ 275 \text {, } \\
€ 275-€ 400 \text {, and }>€ 400 \text {. }\end{array}$ & $\begin{array}{l}30 \text { participants of diverse } \\
\text { demographic backgrounds }\end{array}$ & $\begin{array}{l}3 \text { largest spending categories: } 40 \% \\
\text { clothes/shoes, } 22 \% \text { food, } 9 \% \text { gifts. } \\
\text { Spending on food even across all } \\
\text { levels. Most participants felt they } \\
\text { had little control over their lives. } \\
\text { They appreciated independence in } \\
\text { what they wore and ate. }\end{array}$ \\
\hline $\begin{array}{l}\text { Gold A, 2014; USA, North } \\
\text { Dakota (Fargo) [52] }\end{array}$ & $\begin{array}{c}\text { Evaluate a food safety map as an } \\
\text { educational method with English } \\
\text { language learners. }\end{array}$ & $\begin{array}{l}\text { Adult primary food preparers randomly } \\
\text { assigned to } 1 \text {. Discussion map (tailored to } \\
\text { oral culture learners): principles of food } \\
\text { safety, } 2 \text { h session. } 2 \text {. Cooking: two } 2 \mathrm{~h} \\
\text { classes, basic cooking skills, food safety. } \\
\text { 3. No education. Participants in map and } \\
\text { cooking classes received a food safety } \\
\text { kit, questionnaire. }\end{array}$ & $\begin{array}{l}78 \text { individuals began the study } \\
\text { while } 73 \text { completed the study. }\end{array}$ & $\begin{array}{l}88 \% \text { learned cooking skills from } \\
\text { mothers, } 36 \% \text { from grandmothers, } \\
30 \% \text { from books, } 16 \% \text { from sister, } 8 \% \\
\text { from other family members. >half } \\
\text { cooked for children, } 26 \% \text { for seniors. } \\
\text { Food safety questions answered } \\
\text { more correctly by cooking, } \\
\text { discussion map classes than } \\
\text { control group. }\end{array}$ \\
\hline $\begin{array}{l}\text { Gunnell S, 2015; USA, } \\
\text { Utah [53] }\end{array}$ & $\begin{array}{l}\text { Evaluate if Supplemental Nutrition } \\
\text { Assistance Program (SNAP)-Ed in } \\
\text { English as a Second Language (ESL) } \\
\text { classes at worksite-training reached } \\
\text { eligible population; to pilot } \\
\text { feasibility of food receipts to } \\
\text { evaluate purchasing } \\
\text { before/after classes. }\end{array}$ & $\begin{array}{l}\text { 1-h nutrition lessons in English for } 12 \\
\text { weeks; mandatory training as part of work. } \\
\text { Lessons based on } 2005 \text { USDA Dietary } \\
\text { Guidelines using objectives of SNAP-Ed for } \\
\text { adults, youth. Topics: food safety, food } \\
\text { groups, common acculturation challenges } \\
\text { of packaged/processed foods, budgeting, } \\
\text { shopping, menu planning. }\end{array}$ & $\begin{array}{l}98 \text { recently resettled refugee } \\
\text { participants. } 67 \% \text { completed }>10 \\
\text { nutrition education lessons. } 17 \\
\text { finished the work-site training } \\
\text { program before study completion. } \\
\text { Eligible receipts were collected from } \\
59 \text { participants. }\end{array}$ & $\begin{array}{c}\text { Receipts identified food purchased } \\
\text { by } 25 \text { participants } 1 \text { week prior to } \\
\text { nutrition lessons, } 49 \text { the first } 3 \\
\text { weeks, } 18 \text { the last } 3 \text { weeks, two } 1 \\
\text { week after lessons completed. } 93 \% \\
\text { of receipts reflected use of SNAP } \\
\text { funds, } 15 \% \text { Women, Infants and } \\
\text { Children funds. } 92 \% \text { supermarkets, } \\
59 \% \text { ethnic stores. }\end{array}$ \\
\hline
\end{tabular}


Table 1. Cont.

\section{First Author, Year; Location}

Aim

Present a mixed method eval of a gardening project hosted by churches serving Karen and Bhutanese refugees.
Mixed methods. Survey examined food behaviors, hunger, depression, gardening experience pre- and post-season,

participation in food subsidy programs.

Post-survey, focus groups, interviews with church volunteers.

\section{Participants}

Out of 19 churches

(>1200 refugee/immigrant families), 8 church gardens purposefully sampled based on years of

participation, number of gardeners, languages. 6 focus groups:

$3-10$ people each (48 total). $64 \%$ of gardeners completed both surveys.

Home-based, 1-on-1 counseling for mother

Create, pilot educational materials for home-based counseling of refugee mothers along the

Hashmi A, 2019; Thailand, Mae La refugee camp [55]

Thailand-Myanmar border to improve infant feeding and water, sanitation, and hygiene (WASH) behaviors. with 2-months old healthy term infants; monthly visits from 3-8 months =

counseling, flipbook in basic English,

photos on WASH, exclusive breastfeeding complementary feeding of infants $>6$ months. Infant feeding followed WHO recommendations. for infants $<6$ months, local food for
34 mothers with infants,

$59 \%$ participated in the longitudinal cohort. A total of 132 household

visits were conducted with a median of 7 /household.

Barrier: transportation. Pre-season,

$64 \%$ ate fruit/veg daily vs. $78 \%$ post

$59 \%$ ate $>1$ veg type/day pre-season

vs. $67 \%$ post. Due to lack of

response pre-season, food security questions modified post. $4 \%$

indicated no food in house due to

lack of resources, some went to bed

hungry. $86 \%$ participated in $\geq 1$

food subsidy programs. $92 \% \downarrow$

spending in garden season.

Exclusive breastfeeding: $42 \%$ at

3 months, $65 \%$ at 5 months.

Handwashing: $94 \%$ at baseline

$100 \%$ at $6 / 9$-months. Infants at

6 months fed inadequately, $5 \%$

adequate diet diversity, $10 \%$

appropriate amounts, $0 \%$ minimum acceptable diet; $\uparrow$ to $90 \%, 100 \%, 90 \%$,

by 9 months. Sanitation, safe

disposal of infant stool: $16 \%$ at 6 months, $100 \%$ at 9 months.

All 3 modalities $\uparrow$ quantity and quality of food. Transfers $=\uparrow$ calories, vouchers $\uparrow$ diet diversity.

Randomized design. Curriculum for families, pregnant and lactating women,

children 0-24 months. Transfers if

Compare impact, cost-effectiveness of cash, food vouchers, food

Hidrobo M, 2014; Ecuador, provinces of Carchi and Sucumbíos [56]

transfers on quantity/quality of food consumed. Aimed to influence behavior change, $\uparrow$ knowledge attendance at monthly training. Posters,

flyers: food groups, daily nutritional requirements, sanitation, food preparation, eating a variety and foods that prevent iron,

vit $\mathrm{A}$, calcium, iodine deficiencies.
2087 households had complete food consumption data at baseline and follow-up. $99 \%$ got entire transfers, $88 \%$ on time. All 3 modalities: similar nutrition gains; sig $\uparrow$ Food

Consumption Score (FCS), vouchers and food $\downarrow \%$ of households with poor to borderline FCS. Cash less likely than controls to borrow money. Cash $\$ 42.99 /$ transfer, vouchers $\$ 43.27$ / transfer, food $\$ 58.22 /$ transfer. Cash $=$ least costs (e.g., travel). 
Table 1. Cont.

\begin{tabular}{|c|c|c|c|c|}
\hline First Author, Year; Location & Aim & Intervention & Participants & Results \\
\hline $\begin{array}{l}\text { Hoddinott J, 2020; } \\
\text { Bangladesh, refugee } \\
\text { camps [57] }\end{array}$ & $\begin{array}{l}\text { Examine associations between } \\
\text { electronic food vouchers (e-voucher) } \\
\text { and food rations on nutritional } \\
\text { status of Rohingya children in } \\
\text { Bangladeshi refugee camps. }\end{array}$ & $\begin{array}{l}\text { 2-stage clustered random sampling. } \\
\text { Households assigned General Food } \\
\text { Distribution (GFD): rice, lentils, } \\
\text { micronutrient fortified cooking oil. WHO } \\
\text { standards: linear } \\
\text { growth-length/height-for-age z scores } \\
\text { (HAZ) determine stunting, } \\
\text { thinness-weight-for-height z scores (WHZ) } \\
\text { determine wasting, weight-for-age z scores } \\
\text { (WAZ), mid-upper arm } \\
\text { circumference (MUAC). }\end{array}$ & $\begin{array}{l}2089 \text { Rohingya refugee households } \\
\text { including } 523 \text { children } 6-23 \text { months. } \\
362 \text { children lived in households } \\
\text { that received food rations, } \\
161 \text { e-voucher. } 62 \% \text { of households } \\
\text { received GFD, } 34 \% \text { e-vouchers. } 4 \% \\
\text { that received both were excluded. }\end{array}$ & $\begin{array}{l}36 \% \text { of children in GFD households } \\
\text { were stunted, vs. } 27 \% \text { of children in } \\
\text { e-voucher households. Wasting } \\
\text { measures comparable across groups. } \\
\text { E-vouchers: increase in HAZ, not } \\
\text { stunting. No associations with } \\
\text { weight (WHZ), acute } \\
\text { undernutrition, WAZ, or MUAC. }\end{array}$ \\
\hline $\begin{array}{l}\text { Ibrahim N, 2019; Lebanon, } \\
\text { North of Lebanon and Bekaa } \\
\text { regions [58] }\end{array}$ & $\begin{array}{l}\text { Explore impact of Community } \\
\text { Kitchens (CKs) on food security of } \\
\text { CK workers (CWs) and Syrian } \\
\text { refugee (SR) families. }\end{array}$ & $\begin{array}{c}\text { Exploratory qualitative descriptive } \\
\text { approach. Purposeful and geographical } \\
\text { variation used to recruit } 4 \text { CKs in } 4 \text { areas. } \\
\text { CKs provided both groups with food pots } \\
\text { on regular basis. }\end{array}$ & $\begin{array}{l}\text { CWs: Lebanese or Syrian women } \\
\text { 18-65 years, involved in local CKs } \\
\geq 6 \text { months. SRs: women of } \\
\text { childbearing age with } \geq 1 \text { child, } \\
\text { living in an Informal Tented } \\
\text { Settlement, received or receiving hot } \\
\text { pots from local CK } \geq 6 \text { months. } \\
8 \text { focus groups: } 4 \text { with CWs, } 4 \text { with } \\
\text { SRs. } 15 \text { CWs, } 49 \text { SRs. }\end{array}$ & $\begin{array}{l}\text { CKs had positive impact on food } \\
\text { security, financial, personal, } \\
\text { psychological, societal aspects of } \\
\text { lives. Food pots } \downarrow \text { spending, met } \\
\text { food needs. } 80 \% \text { of SRs }=\text { severe } \\
\text { food insecurity vs. } 40 \% \text { CWs. Some } \\
\text { SRs: choosing families for CKs not } \\
\text { transparent / fair. CKs: } \uparrow \text { variety, } \\
\text { amount of food } \uparrow \text { nutrition, health, } \\
\text { peace of mind. CWs: financial } \\
\text { independence empowering. }\end{array}$ \\
\hline $\begin{array}{l}\text { Inglis K, 2014; Turkey, } \\
\text { refugee camps [59] }\end{array}$ & $\begin{array}{l}\text { Envisioned as efficient, innovative } \\
\text { to let families choose/purchase } \\
\text { diverse, nutritious food with } \\
\text { e-Food Card. }\end{array}$ & $\begin{array}{l}\text { Household assistance on e- cards } \\
\text { bi-monthly with balance at end of month } \\
\text { returned; used in camps, nearby centres. }\end{array}$ & $\begin{array}{c}21 \text { camps, over } 217,000 \text { beneficiaries } \\
\text { in } 45,000 \text { households; } 58 \text { shops. } \\
\text { Most families have children }<5 \text { y } \\
\text { of age. }\end{array}$ & $\begin{array}{c}>90 \% \text { prefer e-cards to hot meals. } \\
>70 \% \text { savings vs. hot meals, } \\
\text { eliminated waste at distributions. } \\
\text { Challenges: } \uparrow \text { prices in shops, } \\
\text { drought. }\end{array}$ \\
\hline $\begin{array}{l}\text { Karama Organization, 2015; } \\
\text { Palestine, Deheishe refugee } \\
\text { camp [60] }\end{array}$ & $\begin{array}{c}\text { Improve refugee food security, } \downarrow \\
\text { dependency on aid, empower } \\
\text { women to } \downarrow \text { stress, } \uparrow \\
\text { physical/mental health. }\end{array}$ & $\begin{array}{l}\text { Gardens }=7 \text { tubes with soil, water system, } \\
\text { net to cover plants, create shade. In winter, } \\
\text { plastic converts to a green house. } \\
\text { Participants provided tools to foster } \\
\text { initiative, creativity, } \uparrow \text { self-esteem. }\end{array}$ & 15 women & $\begin{array}{l}\text { Fresh veg spared limited budgets. } \\
\text { Women felt empowered } \\
\text { contributing to family needs, } \uparrow \\
\text { self-esteem, relieved stress, } \uparrow \text { quality } \\
\text { of life. Green spaces } \uparrow \text { camp } \\
\text { environment. }\end{array}$ \\
\hline
\end{tabular}


Table 1. Cont.

\begin{tabular}{|c|c|c|c|c|}
\hline First Author, Year; Location & Aim & Intervention & Participants & Results \\
\hline $\begin{array}{c}\text { Mannion CA, 2014; Canada, } \\
\text { Calgary Alberta [61] }\end{array}$ & $\begin{array}{l}\text { Assess acceptability of a nutrition } \\
\text { resource developed to help } \\
\text { Sudanese refugee women purchase } \\
\text { healthy foods, navigate grocery } \\
\text { stores. }\end{array}$ & $\begin{array}{l}\text { Grounded theory analysis. Market Guide: } \\
\text { shopping resource to aid Sudanese refugees } \\
\text { with food choices; encourages foods rich in } \\
\text { iron, calcium, vit D; discourages high fat, } \\
\text { low nutrient dense. Booklet: washable, } \\
\text { purse-sized, nutrient-dense foods, serving } \\
\text { sizes (Canada Food Guide), grocery store } \\
\text { map, traditional recipes. Purposive } \\
\text { sampling for focus group, grocery } \\
\text { store visit. }\end{array}$ & $\begin{array}{l}\text { Sudanese adult women in Canada } \\
<1 \text { y. Of } 20 \text { women invited, } \\
8 \text { participated in focus group, } 4 \text { also } \\
\text { attended grocery store visit. } \\
\text { Interviews with } 2 \text { Sudanese } \\
\text { Canadian intake workers, a public } \\
\text { health nurse, center's current } \\
\text { medical director. }\end{array}$ & $\begin{array}{l}\text { Market Guide not well received. } \\
\text { Barriers: language, unknown } \\
\text { foods/stores, limited knowledge. } \\
\text { Mothers' certainty they were doing } \\
\text { well } \downarrow \text { based on ability to feed } \\
\text { family, if children asked for western } \\
\text { food. Often chose traditional over } \\
\text { unfamiliar food, had ingredients } \\
\text { shipped. Families learned from } \\
\text { relatives, friends, community; } \\
\text { children from school, friends. }\end{array}$ \\
\hline $\begin{array}{l}\text { McElrone M, 2020; USA, } \\
\text { mid-sized cities in } \\
\text { Southeastern region [62] }\end{array}$ & $\begin{array}{c}\text { Promote healthful cooking skill } \\
\text { development, enhance family } \\
\text { mealtime, } \uparrow \text { physical activity } \\
\text { through reciprocal role and } \\
\text { behavioral modeling in Sub-Saharan } \\
\text { Africans. }\end{array}$ & $\begin{array}{l}\text { Community-based cultural adaptation of } \\
\text { iCook 4-H: out-of-school child obesity } \\
\text { prevention; Social Cognitive Theory; } \\
\text { 8-session cooking curriculum-diet } \\
\text { acculturation barriers to food security. } \\
\text { Recruitment: local refugee programs, } \\
\text { snowballing. After baseline, dyads } \\
\text { randomly assigned to treatment (2-months } \\
\text { pilot), controls. }\end{array}$ & $\begin{array}{l}10 \text { youth } / \text { mother dyads } \\
\text { ( } 5 \text { treatment, } 5 \text { control) with youth } \\
8-12 \text { years and mothers } \geq 18 \text { years. } \\
\text { Burundian, Congolese } \\
\text { refugee families. }\end{array}$ & $\begin{array}{l}\text { Process eval: positive feedback. } \\
\text { Treatment youth } \uparrow \text { cooking skills, } \\
\text { cooking self-efficacy, eating, setting } \\
\text { healthful goals together as a family; } \\
\downarrow \text { in playing together. Treatment } \\
\text { adults } \uparrow \text { cooking, eating, playing } \\
\text { together, kitchen proficiency, } \\
\text { food security. }\end{array}$ \\
\hline $\begin{array}{l}\text { Millican J, 2019; Iraq, } \\
\text { Kurdistan, Domiz camp [63] }\end{array}$ & $\begin{array}{l}\text { Illustrate benefits of gardening, } \\
\text { need for sustained inclusion in } \\
\text { camp design. }\end{array}$ & $\begin{array}{l}\text { Mixed methods: ground canvassing to } \\
\text { assess the current state of urban } \\
\text { agriculture/gardening in camp, } \\
\text { focus-groups, key informant interviews } \\
\text { with families and individual refugees, and } \\
\text { data about participants' gardens and } \\
\text { whether they had a garden before. }\end{array}$ & $\begin{array}{l}\text { Focus groups: } 1 \text { male, } 1 \text { female. Key } \\
\text { informant interviews: } 10 \text { families, } \\
16 \text { individual refugees from the } \\
2017 \text { garden competition, and data } \\
\text { on } 139 \text { participants. }\end{array}$ & $\begin{array}{l}>50 \% \text { said gardens important for } \\
\text { mental health, wellbeing. Growing } \\
\text { food important, relax, relieve stress; } \\
\text { supplement income, feel happier; } \\
\text { share/trade seeds. Women: } \uparrow \text { social } \\
\text { network, where kids play, find fresh } \\
\text { veg. Motivators: } \uparrow \text { taste, 'clean } \\
\text { water'. Challenges: } \downarrow \text { space, water } \\
\text { (recycle greywater). }\end{array}$ \\
\hline
\end{tabular}


Table 1. Cont.

\begin{tabular}{|c|c|c|c|c|}
\hline First Author, Year; Location & Aim & Intervention & Participants & Results \\
\hline $\begin{array}{l}\text { Mochizuki Y, 2017; Uganda, } \\
\text { Adjumani District [64] }\end{array}$ & $\begin{array}{l}\text { Examine livelihood strategies of } \\
\text { South Sudanese refugees. }\end{array}$ & $\begin{array}{l}\text { Semi-structured interviews with Dinka } \\
\text { people. Refugees given food rations, } \\
25 \mathrm{~m} \times 25 \mathrm{~m} \text { plot of land for food. Rations: } \\
\text { sorghum, unpopular with refugees from } \\
\text { South Sudan, still in grain form, often pay } \\
\text { Ugandans to produce flour. }\end{array}$ & 25 households, mostly women & $\begin{array}{l}\text { Most grew food common in South } \\
\text { Sudan; } 5 \text { households grew sorghum } \\
\text { from rations, sold to host } \\
\text { community; } 4 \text { bred livestock; } \\
13 \text { grew: maize, okra, pumpkin, } \\
\text { sorghum, chard, onion, sesame, } \\
\text { tomato, peanut, cabbage. }\end{array}$ \\
\hline $\begin{array}{l}\text { Ngwenyi E, 2019; Cameroon, } \\
\text { Far North, East, and } \\
\text { Adamaoua regions [65] }\end{array}$ & $\begin{array}{l}\text { Prevent malnutrition in children, } \\
\text { pregnant and lactating women; } \\
\text { ensure nutrition of } \\
\text { nonmalnourished children, already } \\
\text { malnourished = same supplement } \\
\text { in regular moderately acute } \\
\text { malnutrition (MAM) programs. } \\
\text { Target refugees, internally } \\
\text { displaced, hosts. }\end{array}$ & $\begin{array}{l}\text { Super Cereal Plus to children } 6-24 \text { months } \\
\text { to prevent MAM, } 6-59 \text { months to treat } \\
\text { MAM. Social, behaviour change: infant and } \\
\text { young child feeding (IYCF); water, } \\
\text { sanitation, and hygiene; cooking locally } \\
\text { available nutritious foods. Other services: } \\
\text { e.g., immunisation, deworming, malaria } \\
\text { prevention, supplementation, family } \\
\text { planning, capacity-building of } \\
\text { health workers. }\end{array}$ & $\begin{array}{l}\text { Beneficiaries of supplementary } \\
\text { feeding } \uparrow \text { from } 24,000 \text { in } 2015 \text { to } \\
\sim 100,000 \text { in } 2016 / 2017.70 \% \text { of } \\
\text { eligible received SNF, } 90 \% \\
\text { participated in } 66 \% \text { of distributions. } \\
1624 \text { children } 24-59 \text { months referred } \\
\text { to prevention program after } \\
\text { recovery from severe } \\
\text { acute malnutrition. }\end{array}$ & $\begin{array}{l}\text { A monthly surveillance system is } \\
\text { now in place to detect } \\
\text { malnutrition early. }\end{array}$ \\
\hline
\end{tabular}

From 2008 to 2011, food retail shops $\uparrow$ from 7 to 56 , wholesalers from 4 to 8. Quantity, quality of goods, services from aid agencies affected by donor funding, supply chain,

To exemplify the need for informal economies in refugee camps to Oka R, 2011; Kenya, Kakum Refugee Camp [66]

or "refugee camp towns"

Semi-structured interviews, observation of trader-refugee-relief agency interactions. Questions covered role of informa economy in sustaining life at Kakuma, importance for traders, refugees, relief agencies.
78 traders (wholesalers, retailers);

179 refugees; 38 relief workers (UN Agencies, others) distribution = chronic malnutrition

low-quality shelter, education,

training. Frequent shortages due to

droughts, crop failures, budgets,

transport costs. When WFP staff not present, given less. Amount of food

not enough, children hungry, women went without.

Trading $/$ purchasing = dignity power, normalcy. 
Table 1. Cont.

\begin{tabular}{|c|c|c|c|c|}
\hline First Author, Year; Location & Aim & Intervention & Participants & Results \\
\hline $\begin{array}{l}\text { Pavanello S, 2018; Greece } \\
\text { (mainland and islands) [67] }\end{array}$ & $\begin{array}{c}\text { Meet basic } \\
\text { needs, housing, services to refugees, } \\
\text { asylum seekers. }\end{array}$ & $\begin{array}{l}\text { Emergency Support to Integration and } \\
\text { Accommodation program delivered } \\
\text { multipurpose cash assistance. Eval: } \\
\text { primary, secondary data through key } \\
\text { informant interviews, monitoring and eval } \\
\text { data on cash program, other relevant } \\
\text { studies, focus groups with beneficiaries of } \\
\text { multipurpose cash assistance. }\end{array}$ & $\begin{array}{l}\text { Beneficiaries of the Greece Cash } \\
\text { Alliance program totaled 39,233, } \\
\text { including } 6000 \text { refugees the majority } \\
\text { of others asylum seekers. } 43 \% \text { were } \\
\text { Syrians, } 20 \% \text { Iraqis, } 19 \% \text { Afghans, } \\
\text { rest from Iran, Palestine, Pakistan, } \\
\text { Kuwait, others. } 44 \% \text { located in } \\
\text { Athens, } 26 \% \text { on the islands, } 17 \% \text { in } \\
\text { Central Macedonia. }\end{array}$ & $\begin{array}{c}\text { Cash: } \uparrow \text { dignity, sense of safety, } \\
\text { well-being; allowed preferred foods; } \\
\downarrow \text { intra-household tension. } \\
\text { Rations/catered meals described as } \\
\text { inedible, wasted. Women liked } \\
\text { cooking, cleaning-alleviated } \\
\text { boredom. Majority spent cash on } \\
\text { food, amount not enough. Coping } \\
\text { strategies: } \downarrow \text { adult food } \\
\text { quality/quantity; } \downarrow \text { meat, milk, baby } \\
\text { formula; borrow. } \downarrow \text { information on } \\
\text { expenditures, food security. }\end{array}$ \\
\hline $\begin{array}{c}\text { Qleibo E, 2013; Palestine, } \\
\text { Gaza [68] }\end{array}$ & $\begin{array}{l}\text { Cash vouchers targeted nonrefugees } \\
\text { so not reported here. Rabbit raising } \\
\text { program targeted refugees, } \\
\text { nonrefugees to } \uparrow \text { consumption of } \\
\text { fresh meat, provide something to } \\
\text { sell at local markets. }\end{array}$ & $\begin{array}{l}\text { Program targeted those in need, } \\
\text { female-headed households; each received } 4 \\
\text { female and } 1 \text { male rabbit, cages, } 200 \mathrm{~kg} \\
\text { fodder, a veterinary kit, training. Survey } \\
\text { administered } 4 \text { months after receiving } \\
\text { rabbits, 2-years profitability analysis. }\end{array}$ & 286 Gazan households & $\begin{array}{l}98 \% \text { ate, sold, donated meat. } \\
\text { Rabbits tripled in } 1-4 \text { months. } 71 \% \\
\downarrow \text { debt, } 52 \% \text { avoided crisis sales of } \\
\text { assets. Sustainability high: strong } \\
\text { sense of ownership, knowledge, } \\
\text { skills; } \downarrow \text { maintenance, operational } \\
\text { costs; commitment by partners. } \\
2 \text { years after implementation, } \\
50 \% \text { still operating, return } \uparrow>2 x \text {. }\end{array}$ \\
\hline $\begin{array}{l}\text { Sebuliba H, 2014; Jordan, } \\
\text { Amman, Mafraq, Irbid and } \\
\text { Zarqa regions including } \\
\text { Za'atari and Azraq } \\
\text { camps [69] }\end{array}$ & $\begin{array}{l}\text { Introduce Targeted Supplementary } \\
\text { Feeding Programme (TSFP) to treat } \\
\text { moderately acute malnourished } \\
\text { (MAM) Syrian children and women } \\
\text { in camps, urban communities; } \\
\text { ensure access to } \\
\text { age-appropriate food. }\end{array}$ & $\begin{array}{l}\text { To recruit for TSFP, Mid Upper Arm } \\
\text { Circumference (MUAC) used to screen } \\
\text { children under } 5 \text { years, pregnant and } \\
\text { lactating women (PLW), girls. Those } \\
\text { diagnosed with MAM provided } \\
\text { SuperCereal Plus. Follow-up survey. } \\
\text { Blanket complementary food aid } \\
\text { (SuperCereal Plus) provided monthly to all } \\
\text { children 6-23 months in camps. }\end{array}$ & $\begin{array}{l}\text { Za'atari camp: } 223 \text { (168 children, } \\
55 \text { PLW, girls). Local community: } \\
215 \text { (79 children, } 140 \text { PLW, girls) } \\
\text { (numbers reported as published). } \\
\text { Blanket assistance reached an } \\
\text { additional } 8258 \text { children }<5 \text { years in } \\
\text { Za'atari, } 456 \text { in Azraq. }\end{array}$ & $\begin{array}{c}\text { Za'atari: } 68 \% \text { cured, } 23 \% \text { defaulted, } \\
9 \% \text { transferred to outpatient care. } \\
\text { Local community: } 71 \% \text { cured, } \\
22 \% \text { defaulted, } 7 \% \text { nonresponders. } \\
\text { Improved acute malnutrition, GAM } \\
\text { in Za'atri, local community. } \\
\text { Micronutrient deficiencies persist. } \\
\text { Prevalence of anemia: } 50 \% \text { in } \\
\text { children }<5 \text { years, } 64 \% \text { in }<2 \text { years. } \\
\text { Anaemia } 45 \% \text { in girls, women of } \\
\text { reproductive age. }\end{array}$ \\
\hline
\end{tabular}


Table 1. Cont.

\begin{tabular}{|c|c|c|c|c|}
\hline First Author, Year; Location & Aim & Intervention & Participants & Results \\
\hline $\begin{array}{c}\text { Smock L, 2020; USA, } \\
\text { Massachusetts [70] }\end{array}$ & $\begin{array}{c}\text { Improve growth parameters, } \\
\text { anemia in low-income pregnant and } \\
\text { breastfeeding women, children } \\
<5 \text { years. }\end{array}$ & $\begin{array}{l}\text { Special Supplemental Nutrition for Women, } \\
\text { Infants, and Children (WIC): healthy food, } \\
\text { nutrition education, breastfeeding support. } \\
\text { Children divided by } 2-4 \text { visits or } \geq 5 \text { visits. } \\
\text { Measures: hemoglobin; height, weight, } \\
\text { percentiles, z-scores for BMI, } \\
\text { weight-for-height z-scores (acute } \\
\text { undernutrition), height-for-age z-scores } \\
\text { (chronic undernutrition); used } 2000 \text { CDC } \\
\text { growth charts. }\end{array}$ & $\begin{array}{c}62 \% \text { of refugee children }<5 \text { years } \\
\text { who arrived in Massachusetts from } \\
1998-2010 \text { participated in WIC. } \\
779 \text { children with at least } 2 \text { WIC } \\
\text { visits included in analyses. }\end{array}$ & $\begin{array}{l}\text { Of } 73 \text { children with low } \\
\text { weight-for-age at } 1 \text { st visit, } 79 \% \\
\text { recovered by last visit; of } 78 \text { with } \\
\text { low height-for-age, } 77 \% \text { recovered; } \\
\text { of } 36 \text { with low weight-for-height, } \\
78 \% \text { recovered; of } 191 \text { with anemia, } \\
80 \% \text { recovered. Females averaged } \\
3.5 \text { visits until recovery, males } \\
4.3 \text { visits. Those who remain in WIC } \\
\text { may recover better than children } \\
\text { with fewer WIC visits. }\end{array}$ \\
\hline $\begin{array}{c}\text { Stuetz W, 2016; Thailand, the } \\
\text { Western region [71] }\end{array}$ & $\begin{array}{l}\text { Evaluate impact of dietary changes } \\
\text { on micronutrient status in each } \\
\text { trimester of pregnancy. }\end{array}$ & $\begin{array}{l}\text { Micronutrient fortified flour (MFF) as } \\
\text { supplementary food ration to all in Maela } \\
\text { camp, additional oil ration for pregnant and } \\
\text { post-partum women. }\end{array}$ & $\begin{array}{l}533 \text { out of } 764 \text { women }(70 \%) \\
\text { participated in first survey, } 515 \text { out } \\
\text { of } 745(69 \%) \text { in second survey. }\end{array}$ & $\begin{array}{l}\text { MFF } \uparrow \text { daily intakes, particularly vit } \\
\text { A, B-vits, ascorbic acid, zinc, iron; } \\
\text { supplementary oil ration } \uparrow \\
\text { tocopherol intakes. Mean } \\
\text { hemoglobin, high prevalence of } \\
\text { anemia }(60 \%) \text {, iron deficiency }(39 \%) \\
\text { in 3rd trimester constant. }\end{array}$ \\
\hline
\end{tabular}


Table 1. Cont.

\begin{tabular}{|c|c|c|c|c|}
\hline First Author, Year; Location & Aim & Intervention & Participants & Results \\
\hline $\begin{array}{l}\text { Tomkins M, 2019; Iraq, } \\
\text { Kurdistan, Domiz camp [73] }\end{array}$ & Home gardening and tree planting & $\begin{array}{l}\text { Mixed methods: Ground canvassing to } \\
\text { assess current state of urban agriculture, } \\
\text { gardening in Domiz Camp, focus-groups } \\
\text { divided by gender, key informant } \\
\text { interviews with families and refugees from } \\
2017 \text { garden competition, survey data from } \\
\text { competition participants about what } \\
\text { gardens contained and whether they } \\
\text { gardened before. Tools, seeds, } \\
\text { trees provided. }\end{array}$ & $\begin{array}{l}2 \text { focus groups, key informant } \\
\text { interviews: } n=26 \text {, and } 139 \text { surveys. }\end{array}$ & $\begin{array}{l}\text { Key themes: therapeutic value of } \\
\text { gardening; use of space for health, } \\
\text { privacy, community; use of } \\
\text { gardening as release from } \\
\text { frustrations, boredom. Food } \\
\text { gardening widely evident but not } \\
\text { dominant in camps. Food } \\
\text { production ranged from one family } \\
\text { growing a single crop for cash to } \\
\text { micro-allotment gardens of } \\
\text { multiple veg. }\end{array}$ \\
\hline $\begin{array}{c}\text { Trapp M, 2010; USA, } \\
\text { Midwest, South, and West } \\
\text { regions [74] }\end{array}$ & $\begin{array}{l}\text { Food and Nutrition Outreach (FNO) } \\
\text { program to promote communication } \\
\text { of culturally relevant nutrition } \\
\text { information to newcomers to } \\
\text { consider how social meanings, } \\
\text { socioeconomic processes facilitate } \\
\text { changes in food practices. }\end{array}$ & $\begin{array}{l}\text { FNO: visual nutrition flipchart, training } \\
\text { manual, poster, handouts on malnutrition, } \\
\text { healthy eating, shopping, healthy weight, } \\
\text { breast milk, pregnancy nutrition, exercise, } \\
\text { nutrition labels; in } 15 \text { languages. } 16 \text { training } \\
\text { sessions: cultural competency, nutrition, } \\
\text { links to disease, behavioral change, } \\
\text { nutrition outreach tools, action planning. } \\
\text { Eval survey with trainees. Focus groups } \\
\text { with refugees on outcomes: nutrition } \\
\text { knowledge, dietary change, healthy eating. }\end{array}$ & $\begin{array}{c}\text { Training sessions reached } 200 \text { orgs, } \\
453 \text { service providers. Eval surveys } \\
\text { completed by } 89 \text { trainees. } 6 \text { focus } \\
\text { groups conducted with } \\
45 \text { participants (Karen, Burundian, } \\
\text { Congolese, Ethiopian, Burmese, } \\
\text { West African, Hmong youth and } \\
\text { adult refugees). }\end{array}$ & $\begin{array}{l}75 \% \text { of trainers conducted nutrition } \\
\text { outreach after attending training } \\
\text { session. } 75 \% \text { used FNO } \\
\text { flipchart/handouts. Some refugees } \\
\text { and service providers took steps to } \\
\downarrow \text { fat, sugar intake. Positive changes: } \\
\text { food decisions of agency (e.g., } \\
\text { healthier options, milk), behaviour } \\
\text { change of parents at day care } \\
\text { (healthier foods), nutrition } \\
\text { education at schools-help children } \\
\text { use nutrition facts tables, pass } \\
\text { knowledge to parents. }\end{array}$ \\
\hline $\begin{array}{l}\text { Volpato G, 2013; Western } \\
\text { Sahara, northern Mauritania, } \\
\text { and Tindouf [75] }\end{array}$ & $\begin{array}{l}\text { Assess how role of ethnobiological } \\
\text { knowledge and practices for } \\
\text { refugees' agency, through use and } \\
\text { commodification of desert truffles, } \\
\text { affects Sahrawi refugees of } \\
\text { Western Sahara. }\end{array}$ & $\begin{array}{l}\text { Semi-structured and retrospective } \\
\text { interviews; "walk in the woods" approach } \\
\text { in northern liberated territories with } \\
\text { knowledgeable truffle harvesters (nomads } \\
\text { and refugees). }\end{array}$ & $\begin{array}{c}28 \text { semi-structured interviews, } \\
8 \text { retrospective interviews. The } \\
\text { "walk in the woods" approach with } \\
4 \text { informants. }\end{array}$ & $\begin{array}{l}\text { Truffles: delicacy, complementary, } \\
\text { medicinal, emergency food. } \\
\text { Resources for harvesting: } \\
\text { knowledge (traditions taught by } \\
\text { older refugees), access to territories, } \\
\text { capital for commercial harvesting. } \\
\text { Commodification generates income, } \\
\text { recovers traditional knowledge; } \uparrow \\
\text { harvesting, } \\
\text { competition; unsustainable. }\end{array}$ \\
\hline
\end{tabular}


Table 1. Cont.

\begin{tabular}{|c|c|c|c|c|}
\hline First Author, Year; Location & Aim & Intervention & Participants & Results \\
\hline $\begin{array}{c}\text { Volpato G, 2014; Western } \\
\text { Sahara [76] }\end{array}$ & $\begin{array}{l}\text { Understand Sahrawi refugees' } \\
\text { agency for recovery, adaptation of } \\
\text { traditional subsistence, other } \\
\text { material, cultural practices. Focused } \\
\text { on Ch. 2: camel husbandry-camels, } \\
\text { food security (Ch. } 7 \text { on truffles } \\
\text { examined in published article } \\
\text { (Volpato, 2013). }\end{array}$ & $\begin{array}{l}\text { Mixed methods analysed Sahrawi refugees' } \\
\text { recovery, adaptation of traditional practices } \\
\text { in desert environment including camel } \\
\text { husbandry. Surveys, interviews, focus } \\
\text { groups, observation, 'walk-in-the-woods' } \\
\text { approach, free-listings, ethnobiological } \\
\text { voucher specimen collection. }\end{array}$ & $\begin{array}{l}\text { Open interviews: } 44 \text { camel owners, } \\
30 \text { nomads. Semi-structured } \\
\text { interviews: } 36 \text { refugee and nomadic } \\
\text { camel owners (from open } \\
\text { interviews). Focus groups: } 5 \text { with } \\
\text { refugee camel owners and } \\
\text { older refugees. }\end{array}$ & $\begin{array}{l}\text { Camel husbandry }=\text { traditional } \\
\text { staple foods where agriculture } \\
\text { barely possible; hunting, gathering } \\
\text { limited. } \uparrow \text { success if own vehicle, } \\
\text { GPS, satellite phones, new wells, } \\
\text { pumps, tanker trucks. Sahrawi } \\
\text { camp: positive impact on regional } \\
\text { economies acting as hubs to sell } \\
\text { animals. Revitalised cultural } \\
\text { significance of camel as symbol of } \\
\text { ethnic identity. }\end{array}$ \\
\hline $\begin{array}{c}\text { Wilson A, 2012; Australia, } \\
\text { Victoria [77] }\end{array}$ & $\begin{array}{l}\text { Determine standard meal weight } \\
\text { using evidence-based nutrition } \\
\text { principles, method to convert food } \\
\text { collected by SecondBite into } \\
\text { correctly defined standard, } \\
\text { nutritionally acceptable meals, meet } \\
30 \% \text { nutritional needs of avg adult. }\end{array}$ & $\begin{array}{l}\text { Cross-sectional. Used food collection } \\
\text { database over } 3 \text { months. Observation, } \\
\text { probing on social process of food collection, } \\
\text { management, distribution. Used Australian } \\
\text { Guide to Healthy Eating (AGHE) manual to } \\
\text { develop definition of standard meal, assess } \\
\text { nutritional quality at } 2 \text { charities; calculated } \\
\text { using FoodWorks software. }\end{array}$ & Adults $19-60$ years & $\begin{array}{l}\text { \% nutritional requirements } \\
\text { 20:30:30:20, for breakfast, lunch, } \\
\text { dinner, snacks. Total weight } 30 \% \text { of } \\
\text { AGHE's recommendations } \sim 500 \mathrm{~g}, \\
\text { (10\% breads, } 26 \% \text { veg, } 26 \% \text { fruit, } \\
25 \% \text { dairy, } 9 \% \text { meat and alternatives, } \\
4 \% \text { other); }=30-36 \% \text { energy, } 60-65 \% \\
\text { protein, } 64 \% \text { vit } C, 76 \% \text { calcium, } 38 \% \\
\text { iron for men, } 17 \% \text { iron for women. }\end{array}$ \\
\hline $\begin{array}{l}\text { World Food Programme, } \\
\text { 2014; Mozambique, Maputo } \\
\text { and } 4 \text { Districts in Gaza } \\
\text { Province; } 3 \text { Districts in } \\
\text { Zambezia Province; and } 1 \\
\text { refugee camp in Nampula } \\
\text { Province (Maratane } \\
\text { camp) [78] }\end{array}$ & $\begin{array}{c}\text { Support populations who become } \\
\text { transiently food insecure as a result } \\
\text { of recurring seasonal shocks to: save } \\
\text { lives, protect livelihoods in } \\
\text { emergencies, restore/rebuild lives, } \\
\text { livelihoods in post-conflict, } \\
\text { post-disaster or transition situations; } \\
\text { strengthen capacities of countries to } \\
\downarrow \\
\downarrow \text { hunger. }\end{array}$ & $\begin{array}{l}\text { General Food Distribution (GFD) to } \\
\text { disaster-affected households, refugees at } \\
\text { Maratane; Food for Assets (FFA) to } \\
\text { implement activities to rehabilitate assets, } \\
\text { maintain food security; capacity } \\
\text { development for gov stakeholders. Eval: } \\
\text { mixed methods. Qualitative: in-depth } \\
\text { structured, semi-structured interviews with } \\
\text { WFP staff, stakeholders; focus groups (by } \\
\text { gender) with beneficiaries, nonbeneficiaries, } \\
\text { stakeholders; direct observation. }\end{array}$ & $\begin{array}{l}8000 \text { refugees in Maratane (only } \\
\text { location where humanitarian aid } \\
\text { offered, interviews for refugee } \\
\text { status conducted); } 2805 \text { asylum } \\
\text { seekers, } 718 \text { refugees on outskirts. } \\
\text { Eval: document review, session with } \\
\text { CO, stakeholders' workshop, } \\
2 \text { debriefs to present preliminary } \\
\text { findings, } 110 \text { stakeholder interviews, } \\
33 \text { focus groups with } 205 \text { women, } \\
185 \text { men. }\end{array}$ & $\begin{array}{l}\text { Refugees in camp can travel out for } \\
\text { work if registered. Food rations } \\
\text { appropriate since markets not fully } \\
\text { functioning. FFA did not } \\
\text { appropriately target most in need. } \\
\text { National capacity for contingency } \\
\text { planning, food security, emergency } \\
\text { assessment } \uparrow \text {; sustainability still a } \\
\text { concern. Effective, efficient supply } \\
\text { chain management strongest asset } \\
\text { of program, saving lives, } \uparrow \text { food } \\
\text { security, exceeding GFD targets, } \\
\text { timely delivery. Underfunding } \\
\text { affected FFA targets in } 2012, \text { GFD } \\
\text { tonnage targets in } 2013 \text {. }\end{array}$ \\
\hline
\end{tabular}


Table 1. Cont.

\section{First Author, Year; Location}

Syria: Targeted General Food Distribution

(GFD) as household in-kind rations, blanket

in-kind supplementary feeding for children 6-59 months, vouchers for

pregnant/lactating women, in-kind school-feeding. Lebanon: GFD to eligible

Provide food assistance to

World Food Programme, 2015a; Jordan, Lebanon, Iraq Turkey and Egypt [5]

vulnerable households whose food nutrition security adversely affected by civil unrest in Syria, to save lives, protect livelihoods in emergencies. out of camp refugees, returning Lebanese as 1-month in-kind parcels then vouchers. Turkey: GFD to all refugees in camps as

Response scaled up quickly, assisting 4.25 M people in Syria, $2 \mathrm{M}$ refugees across the region. WFP reached $88 \%$ of target in Egypt, $98 \%$ of all registered refugees in Jordan. Eval: 259 (55\% women) interviews,

47 focus groups, 32 responses to stakeholder questionnaire (majority from Syria). In Jordan, Turkey, eval further considered views from refugees in and outside camps; data from host communities collected where feasible in Lebanon, Turkey. ouchers in 1 camp, in-kind school feeding in 2 camps. Egypt: GFD as vouchers to eligible out of camp refugees, Palestinians from Syria. Fieldwork in Jordan, Lebanon Turkey; remote collection in Egypt, Iraq, Syrian Arab Republic. Interviews, focus groups, stakeholder questionnaire.
Issues: timely baseline data,

inconsistent staffing, inadequate oversight, WFPs proximity to Syrian gov. New refugees in Jordan $(12 \%)$ and Lebanon (16\%) had poor FCS, vs. $4 \%$ and $3 \%$ of refugees on aid. Acceptable FCS on arrival 50\% in Lebanon, improved to 78-98\% (all countries). Focus groups: importance of food aid-main source of income for purchasing food. Most common food coping strategy: less preferred/less expensive food. Aid coping strategies. Benefits to local

economies, refugee-host

relationships improved with e-vouchers. In-kind food usually on time, vouchers subject to delays.

Vouchers periodically resulted in $>$ normal market prices. WFP built complex transport/logistics network to prevent inappropriate relationships with armed groups by rotating companies, drivers, routes. 
Table 1. Cont.

\begin{tabular}{|c|c|c|c|}
\hline First Author, Year; Location & Aim & Intervention & Participants \\
\hline $\begin{array}{c}\text { World Food Programme, } \\
\text { 2015b; Iran, provinces of } \\
\text { Fars, Kerman, } \\
\text { Khorasan-e-Razavi, Markazi, } \\
\text { West Azerbaijan, and } \\
\text { Yazd [79] }\end{array}$ & $\begin{array}{l}\text { Improve food consumption of } \\
\text { vulnerable refugees, } \uparrow \text { access to } \\
\text { education and human capital } \\
\text { development for refugee } \\
\text { girls, youths. }\end{array}$ & $\begin{array}{l}\text { General Food Distribution (GFD) with } \\
\text { target approach-2 levels of household } \\
\text { vulnerability, } 2 \text { food rations; School } \\
\text { Feeding-take home rations to women } \\
\text { teachers, girls in primary/secondary } \\
\text { schools; Food for Training- take home } \\
\text { rations to trainees. Midterm eval: } \\
\text { secondary data, semi-structured interviews } \\
\text { of focus groups and individual households, } \\
\text { interviews with stakeholders, observation, } \\
\text { internal/external debriefings. }\end{array}$ & 30,000 refugees and 200 teachers. \\
\hline
\end{tabular}

World Food Programme,

2016a; Ethiopia, Gambella, Afar, Tigray (Shire)

BenishangulGumuz (Assosa)

and Somali (Dolo Ado and

Jijiga) [80]
Assess previous operation's transition period, performance of current operation to ensure informed decisions, future design strategies.
General food distribution, school feeding (SF), blanket and targeted supplementary feeding, livelihood support. Eval: lit review, observation, in-depth semi-structured interviews. > half of country's households are food insecure as defined as per capita access to calories.
130 key informant interviews,

35 focus groups with 401 participants (207 female, 194 male).
Targeting process lacked accuracy (selection criteria), participation. Most aid through GFD, $\downarrow$ support for livelihood strategies. Targets reached despite operational limits (i.e., import constraints due to sanctions on Iran) $=\operatorname{sig} \downarrow$ deliveries than planned. Lack of measure,

unreliable indicators $=$ difficult to analyse. Food consumption maintained or $\uparrow$ with aid for refugees in settlements.

Supplementary feeding reached vulnerable children, mothers; global acute malnutrition still $\uparrow$. Women, children collect firewood- $\uparrow$ risk of ender-based violence. Food ration $89 \%$ to $95 \%$ of target, cash $89 \%$ to $>100 \%$. Household diet diversity, food consumption met targets. Food distributions fair, smaller households (women) at disadvantage. SF 44 to $79 \%$ of target, satisfactory, appreciated. School retention exceeded targets. Cash transfers: $\downarrow$ sale of food aid, $\uparrow$ choice, flexibility; somewhat $\uparrow$ food eaten, $\uparrow$ empowerment, dignity. Cash, biometrics $\downarrow$ fraud, need to sell rations. 
Table 1. Cont.

Liberia affected by Ebola, impacted program delivery, delayed repatriation. Gaps, inaccuracies found in data. Effectiveness limited-all activities except GFD suspended ( $\downarrow$ funding). GFD

prioritized in camps, (an approach

General Food Distribution (GFD), School Feeding (SF), Food for Assets (FFA) to $\uparrow$ access to markets, enhance agriculture

World Food Programme, 2016b; Liberia, Maryland, Grand Gedeh, and Nimba counties [81]
FFA objective: to protect livelihoods, create assets for vulnerable host populations, refugees living in host communities. School Feeding objective: support enrolment, retention in school production by providing each participan months, targeted supplementary feeding (TSF) for Moderate Acute Malnutrition (MAM) in host communities, Stunting

Prevention Programme in host

communities and refugee camps. Eval:

document reviews, key informant

interviews, observations. Standardised

Expanded Nutrition Surveys assessed nutrition status.
Food aid to 100,136. Eval:

370 persons interviewed during key informant interviews and

35 disaggregated focus groups

(65 community members and

137 refugees in camps and host

communities). SF in 3 primary

schools in 3 refugee camps assisting 7694 children. that promoted self-sufficiency,

addressing vulnerable groups would have $\downarrow$ dependency on external aid). $73 \%$ planned GFD delivered maintaining nutritional status, few outcome targets met. For

children 6-59 months, acute malnutrition $=$ acceptable $\mathrm{WHO}$ levels, chronic malnutrition critical prevalence of anaemia $\downarrow 78 \%$ to $67 \%$, TSF not successful; SPP should not have been suspended. SF $=77 \%$ of target; aimed to $\uparrow$ enrolment by $6 \% / y$, inconclusive. FFA activities benefited Liberians, refugees in host communities. $20-30 \%$ of households had acceptable food security,

$20-40 \%$ of rations sold to purchase other food. 
Table 1. Cont.

Implementation efficient, effective including supply chain; no funding interruptions. Commodities $=$ good quality, maize and beans for GFD procured locally, $\sim 10 \%$ purchased

Meet food/nutrition needs of refugees, returnees; treat acute

World Food Programme, 2016c; Rwanda, Gihembe,

Mahama, Kigeme,

Nyabiheke, and Kiziba

camps, and Bugesera and

Nkamira transit centres [82]

malnourished children 6-59 months;

prevent chronic malnutrition in

children 6-23 months; prevent

malnutrition while $\uparrow$ adherence to

drug protocols of people living with

HIV on antiretroviral treatment and

patients; $\uparrow$ access, quality of

education/health facilities in camps.
Food aid through general food distribution (GFD) or cash-based transfers (CBT), and nutrition and school feeding (SF) programs.

SF: 1 meal/school day to children at

13 primary/secondary schools, in camps or host communities. Midterm eval: mixed methods including a document review,

$$
\text { observation, in-depth }
$$

structured/semi-structured interviews with key informants, focus groups with refugees,
GFD 81,593, CBT 49,816. Preventive feeding 19,700 children $<5$ years, 8458 pregnant and lactating women.

Curative feeding 3255 children

$<5$ years, 1224 medical cases. SF

34,731 primary children, including 8900 in host community. Eval:

170 key informant interviews,

29 focus groups (223 women, 105 men) plus 40 random

impromptu focus groups. from small farmers. Distribution

facilities well run. Households with

poor food security remained steady

acceptable food security $\uparrow$ to $77 \%$.

Average coping strategies index

scores $\downarrow$ from 11.4 to 9.7, meeting

targets. Dietary diversity was below expectations in cash camps (4.24),

ahead of food camps (4.07).

Nutrition program $\downarrow$ global acute

malnutrition in Mahama, stabilised malnutrition for pregnant women

children. SF was a significant, well-managed activity. Few

livelihood opportunities; plans for grinding mill, gardening, rabbit production in place. 
Table 1. Cont.

Coverage $\uparrow$, but below target.

Rations sold as sorghum unfamiliar, disliked, to purchase other items.

Traders $\uparrow$ price; delays in

CBTs, called Bamba Chakula (Swahili for 'get your food') -introduced to all registered refugees in camps in response to $\downarrow$ dietary

Cash Based Transfers (CBT) scaled diversity, reselling in-kind aid at a loss.

World Food Programme

2018a; Kenya, Kakuma

camp, Kalobeyei settlement and Dadaab camp [83] up to $\uparrow$ cost effectiveness of food aid in Kenyan Refugee Operations to develop a model that determines effective, efficient mix between food aid, CBT.
CBTs: monthly e-vouchers via SIM cards to

buy food through contracted traders. CBTs substituted cereal rations, began by replacing $10 \%$, $\uparrow$ to $30-50 \%$. Eval: mixed methods including quantitative surveys, in-depth interviews, focus groups, key informant interviews. Data was gender disaggregated. Comparison due to lack of control group.
Food, CBT to all refugees 146,7682 Kakuma, 38,170 Kalobeyei, 235,2964 Dadaab). Some refugees living in camps 25 years.

Quantitative surveys administered to 542 households in Kakuma, 545 households in Kalobeyei; 230 traders, 626 households from host and nonhost communities. Refugees from South Sudan $56.4 \%$, Somalia 20\%, Ethiopia 5.6\%. disbursements $=$ credit purchases, mostly female households = loyalty, indebted to traders. Kakuma $=\downarrow$

food security, diet diversity; $\downarrow$ nutritious foods than Kalobeyei due to $\uparrow$ transfer value in Kalobeyei, ration cuts, delayed disbursements, $\downarrow$ purchasing power in Kakuma. Kalobeyei $=\uparrow$ severe hunger, asset poverty, $\downarrow$ livelihood opportunities, worse gender equality. Female households worse across indicators. Positive impacts on livelihoods, food security in host vs. distan communities. CBT more cost-efficient than transfers. 
Table 1. Cont.

Financial limitations $\downarrow$ diversity, nutrition of food aid. Most nutrition food security outcomes sig $\uparrow$.

Prevalence of acute and chronic

Improve food consumption of most vulnerable refugees through food aid, to $\downarrow$ acute malnutrition,

World Food Programme 2018b; Algeria, 5 refuge camps near Tindouf [84] anaemia in children $<5$ years,

pregnant and lactating women

(PLW) through targeted nutrition

interventions; to maintain

enrolment/retention of children.
Nutrition components used General Food Distribution (GFD), prevention, treatment of undernutrition, anaemia among children $<5$ years, PLW, School Feeding (SF). Eval used mixed methods: key informant interviews, focus groups, field visits, storytelling, Photovoice.
Monthly planned GFD rations targeted 125,000. Targeted

Supplementary Feeding Programme 1800 children/month

1000-6000 PLW/month. Preventive component 13,250 children/month prevention of anaemia $6360 \mathrm{PLW} / \mathrm{month}$, SF 32,500 children/month. Numbers may not be accurate due to data discrepancies. malnutrition $\downarrow$ in children, below emergency levels, underweight

residual in women. $\mathrm{Sig} \uparrow$ of

overweight, obesity, metabolic risk

contributing to double burden of undernutrition and obesity. Those who could afford it purchased food to complement rations. Photovoice $=$ valued foods not often distributed. Satisfied with aid, request $\uparrow$ quantity/quality, regular distribution. Diets did not mee requirements for calcium, iron, niacin, vit $C$, vit $A$. Acceptable food security $\uparrow 77 \%$ to $93 \%$. 
Table 1. Cont.

CBTs, shift from nutrition treatment

to prevention = positive. $\uparrow$ efficiency with CBTs, mobile vulnerability analysis, mapping for data

collection in areas with restricted

General food assistance (GFA) introduced then cash-based transfers (CBTs). Nutrition interventions treated malnutrition through

Improve resilience to address

World Food Programme, 2018c; Cameroon, the Far

North, North, East and Adamaoua regions [85] vulnerability towards

climatic hazards. food insecurity, household targeted supplementary food to children $<5$ years, pregnant and lactating women

GFA $1,268,998$ (104\% of target), nutrition activities 1,879,003 $(86 \%$ of target), school feeding 91,728 (25\% of target), FFA 397,648 ( $55 \%$ of complement for children 6-23 months.

Food for assets (FFA) for refugees, host populations with moderate food insecurity.

Food by prescription for malnourished people living with HIV, receiving anti-retroviral therapy. Schoo feeding implemented. target), Food by prescription 3819

(

Nigeria, Central African Republic. access. Funding shortfalls,

delays $=\downarrow$ rations, temporary suspensions of distributions, cessation of school meals. Sustainability of FFA activities limited. Food insecurity in Cameroon $\uparrow$ to $10 \%$. 32\% of children $<5$ years chronically malnourished $13 \%$ severely stunted. Chronic and acute malnutrition high in N., E.

Cameroon, improved in the Far N., N., Adamaoua when WFP provided support. Malnutrition deteriorated in E. With $\uparrow$ refugees from Central African Republic 
Table 1. Cont.

\begin{tabular}{|c|c|c|c|c|}
\hline First Author, Year; Location & Aim & Intervention & Participants & Results \\
\hline $\begin{array}{l}\text { Wtsadik M, 2011; Ethiopia, } \\
\text { Shimbelba, Awbarre, and } \\
\text { Kebribeyah refugee } \\
\text { camps [86] }\end{array}$ & $\begin{array}{c}\uparrow \text { availability of veg, eggs at } \\
\text { household level, thereby } \uparrow \\
\text { micronutrient status of } \\
\text { vulnerable refugees. }\end{array}$ & $\begin{array}{l}\text { Multi-storey gardens (MSG), poultry } \\
\text { provided to } 3 \text { camps. Oil cans filled with } \\
\text { rocks in } 50 \mathrm{~kg} \text { cereal bags with holes in top, } \\
\text { sides. Seeds planted on top, thinned out, } \\
\text { inserted in sides. Required } 5 \text { L water } \\
2 \text { / day-recommended greywater. Each } \\
\text { household encouraged to build } 5 \text { MSGs, } \\
\text { provided } 3 \text { poultry ( } 1 \text { male, } 2 \text { females). } \\
\text { Targeted family members with anaemia or } \\
\text { malnutrition, large female-headed families, } \\
\text { people with HIV / AIDS. Eval: } \\
\text { questionnaires on veg consumption, veg } \\
\text { sold, \% rations sold to buy veg, water use, } \\
\text { egg consumption. }\end{array}$ & $\begin{array}{l}167 \text { households in each of } 3 \text { camps. } \\
\text { Eval: } 50 \text { households (random } \\
\text { selection). Focus groups: } \\
\text { 15-20 households (random } \\
\text { selection) not included in household } \\
\text { survey and } 5 \text { households who were } \\
\text { not one of the } 167 \text { beneficiary } \\
\text { households. }\end{array}$ & $\begin{array}{l}\text { Compared to backyard gardens, } \\
\text { MSGs needed } \downarrow \text { water, veg grew } \\
\text { faster, } 2 \text { harvests possible. Refugees } \\
\text { acquired new skills, diverse meals, } \\
\text { shared produce, less likely to sell } \\
\text { rations for veg. At eval, chickens too } \\
\text { small to lay eggs in } 2 \text { camps, but in } \\
1 \text { camp, 35\% of participants ate } \\
\sim 7.5 \text { eggs/week. Project well } \\
\text { accepted, requested by nonpilot } \\
\text { households; allowed refugees to } \\
\text { choose what to plant/eat, gave } \\
\text { sense of dignity, well-being. Some } \\
\text { refugees trying to duplicate MSG on } \\
\text { their own. Poultry not } \\
\text { recommended: chickens ate } \\
\text { produce, } \uparrow \text { cost, time. }\end{array}$ \\
\hline
\end{tabular}




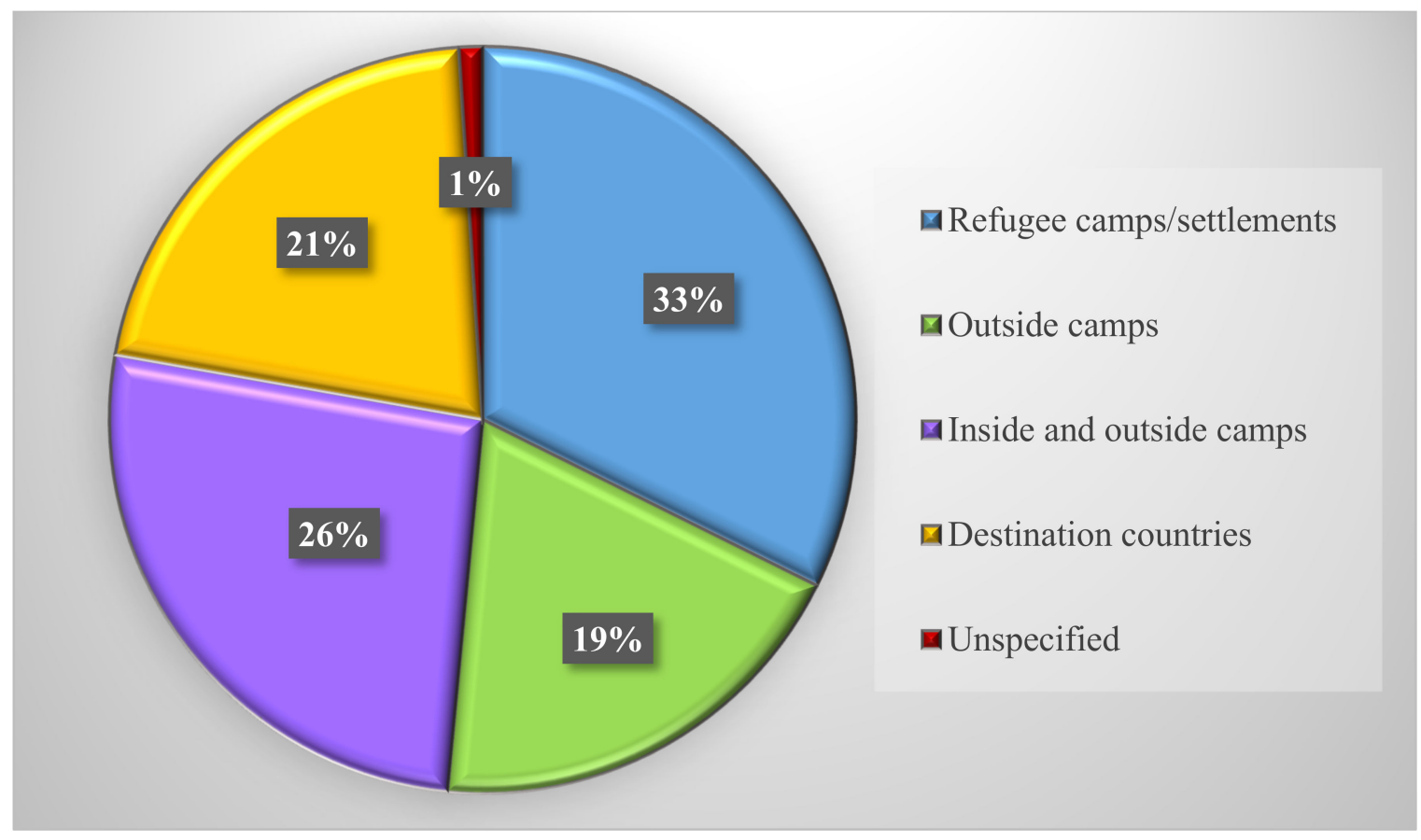

Figure 2. Percent of articles by location.

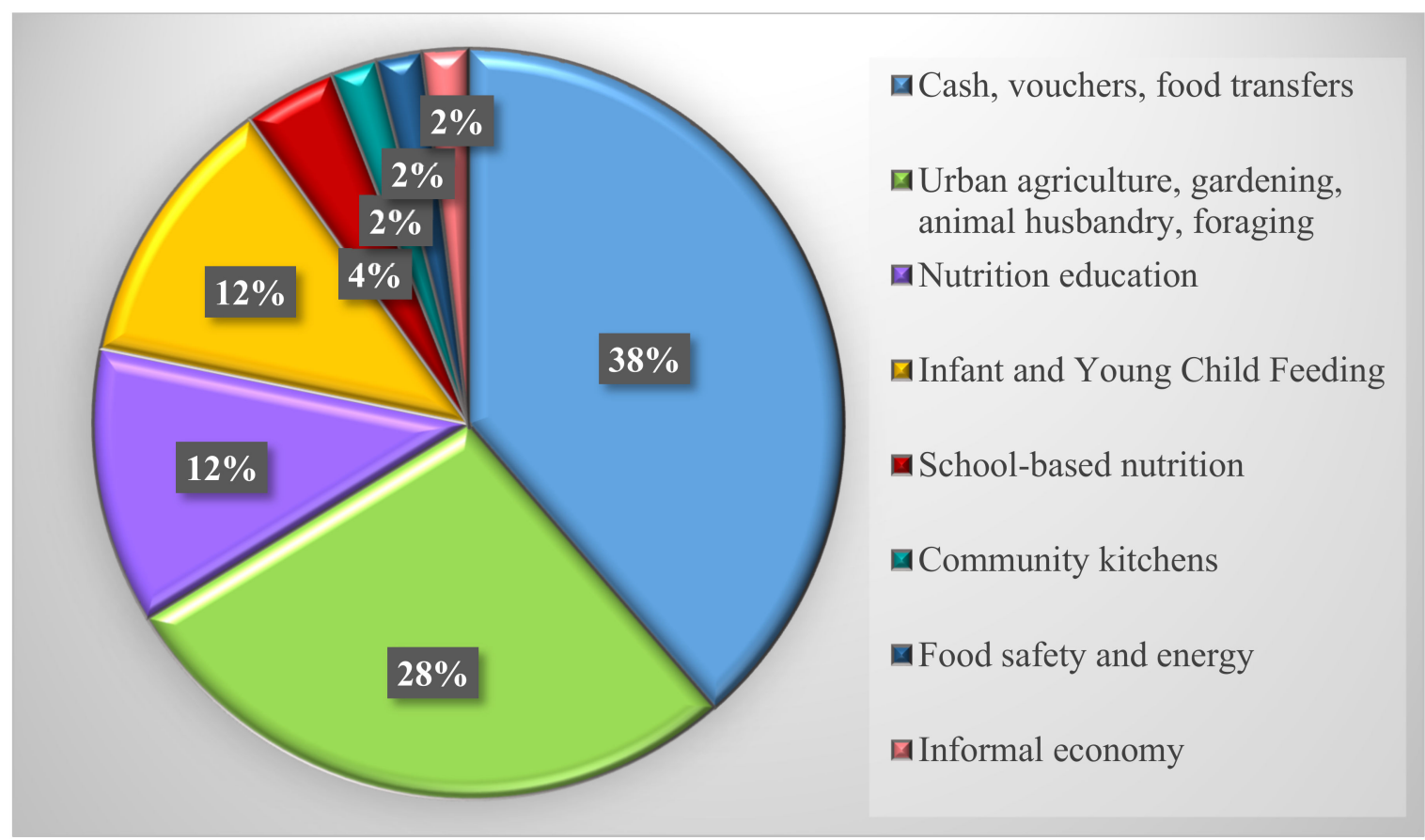

Figure 3. Percent of articles by intervention type. 
Table 2. Themes of eligible studies.

\begin{tabular}{|c|c|c|c|c|c|}
\hline First Author, Year & Location & Target Population & Intervention Type & $\begin{array}{l}\text { Considers } \\
\text { Gender }\end{array}$ & $\begin{array}{l}\text { Food Security } \\
\text { Measure }\end{array}$ \\
\hline $\begin{array}{c}\text { World Food } \\
\text { Programme, 2018b [84] }\end{array}$ & $\begin{array}{l}\text { Algeria: Refugee } \\
\text { camps/informal } \\
\text { settlements }\end{array}$ & Refugees only & $\begin{array}{l}\text { Mixed cash, } \\
\text { vouchers, food } \\
\text { transfers }\end{array}$ & No & $\begin{array}{l}\text { Dietary Diversity } \\
\text { Score, Food } \\
\text { Consumption } \\
\text { Score, Coping } \\
\text { Strategies Index }\end{array}$ \\
\hline Wilson A, 2012 [77] & $\begin{array}{l}\text { Australia: } \\
\text { Destination } \\
\text { country }\end{array}$ & $\begin{array}{l}\text { Refugees and host } \\
\text { communities }\end{array}$ & $\begin{array}{c}\text { Nutrition } \\
\text { education } \\
\text { programming }\end{array}$ & No & No \\
\hline Gichunge C, 2014 [49] & $\begin{array}{l}\text { Australia: } \\
\text { Destination } \\
\text { country }\end{array}$ & Refugees only & $\begin{array}{l}\text { Urban agriculture, } \\
\text { animals, foraging }\end{array}$ & No & No \\
\hline Hoddinott J, 2020 [57] & $\begin{array}{l}\text { Bangladesh: } \\
\text { Refugee } \\
\text { camps/informal } \\
\text { settlements }\end{array}$ & Refugees only & $\begin{array}{l}\text { Mixed cash, } \\
\text { vouchers, food } \\
\text { transfers }\end{array}$ & Yes & No \\
\hline Ngwenyi E, 2019 [65] & $\begin{array}{l}\text { Cameroon: Inside } \\
\text { and outside camps }\end{array}$ & $\begin{array}{l}\text { Refugees and host } \\
\text { communities }\end{array}$ & $\begin{array}{l}\text { IYCF * and } \\
\text { pregnancy }\end{array}$ & Yes & No \\
\hline $\begin{array}{c}\text { World Food } \\
\text { Programme, 2018c [85] }\end{array}$ & $\begin{array}{l}\text { Cameroon: Inside } \\
\text { and outside camps }\end{array}$ & $\begin{array}{l}\text { Refugees and host } \\
\text { communities }\end{array}$ & $\begin{array}{l}\text { Mixed cash, } \\
\text { vouchers, food } \\
\text { transfers }\end{array}$ & Yes & $\begin{array}{l}\text { Dietary Diversity } \\
\text { Score, Food } \\
\text { Consumption } \\
\text { Score, Coping } \\
\text { Strategies Index }\end{array}$ \\
\hline Mannion CA, 2014 [61] & $\begin{array}{l}\text { Canada: } \\
\text { Destination } \\
\text { country }\end{array}$ & Refugees only & $\begin{array}{c}\text { Nutrition } \\
\text { education } \\
\text { programming }\end{array}$ & Yes & No \\
\hline $\begin{array}{c}\text { Food and Agriculture } \\
\text { Organization of the } \\
\text { United Nations, } \\
2020[47]\end{array}$ & $\begin{array}{l}\text { DR Congo: } \\
\text { Outside camps }\end{array}$ & $\begin{array}{l}\text { Refugees and host } \\
\text { communities }\end{array}$ & $\begin{array}{l}\text { Urban agriculture, } \\
\text { animals, foraging }\end{array}$ & Yes & No \\
\hline Hidrobo M, 2014 [56] & $\begin{array}{l}\text { Ecuador: Outside } \\
\text { camps }\end{array}$ & $\begin{array}{l}\text { Refugees and host } \\
\text { communities }\end{array}$ & $\begin{array}{l}\text { Mixed cash, } \\
\text { vouchers, food } \\
\text { transfers }\end{array}$ & Yes & $\begin{array}{c}\text { Food } \\
\text { Consumption } \\
\text { Score }\end{array}$ \\
\hline $\begin{array}{c}\text { World Food } \\
\text { Programme, 2016a [80] }\end{array}$ & $\begin{array}{l}\text { Ethiopia: Inside } \\
\text { and outside camps }\end{array}$ & Refugees only & $\begin{array}{l}\text { Mixed cash, } \\
\text { vouchers, food } \\
\text { transfers }\end{array}$ & Yes & $\begin{array}{c}\text { Per capita access to } \\
\text { calories }\end{array}$ \\
\hline WTsadik M, 2011 [86] & $\begin{array}{l}\text { Ethiopia: Refugee } \\
\text { camps/informal } \\
\text { settlements }\end{array}$ & Refugees only & $\begin{array}{l}\text { Urban agriculture, } \\
\text { animals, foraging }\end{array}$ & Yes & No \\
\hline Goh J, 2017 [51] & $\begin{array}{l}\text { Germany: } \\
\text { Destination } \\
\text { country }\end{array}$ & Refugees only & Cash & No & No \\
\hline Dunlop K, 2018 [23] & $\begin{array}{l}\text { Greece: Inside and } \\
\text { outside camps }\end{array}$ & Refugees only & Cash & No & $\begin{array}{l}\text { Questions on } \\
\text { challenges in } \\
\text { accessing } \\
\text { shops/markets; } \\
\text { travel time to } \\
\text { nearest } \\
\text { market/shop; } \\
\text { travel costs; } \\
\text { food/nonfood } \\
\text { items in the last } 2 \\
\text { months. }\end{array}$ \\
\hline
\end{tabular}


Table 2. Cont.

\begin{tabular}{|c|c|c|c|c|c|}
\hline First Author, Year & Location & Target Population & Intervention Type & $\begin{array}{l}\text { Considers } \\
\text { Gender }\end{array}$ & $\begin{array}{l}\text { Food Security } \\
\text { Measure }\end{array}$ \\
\hline Pavanello S, 2018 [67] & $\begin{array}{l}\text { Greece: Inside and } \\
\text { outside camps }\end{array}$ & Refugees only & Cash & Yes & No \\
\hline $\begin{array}{c}\text { World Food } \\
\text { Programme, 2015b [79] }\end{array}$ & $\begin{array}{l}\text { Iran: Outside } \\
\text { camps }\end{array}$ & Refugees only & $\begin{array}{l}\text { Mixed cash, } \\
\text { vouchers, food } \\
\text { transfers }\end{array}$ & Yes & $\begin{array}{c}\text { Food } \\
\text { Consumption } \\
\text { Score and Diet } \\
\text { Diversity Score }\end{array}$ \\
\hline Tomkins M, 2019 [73] & $\begin{array}{l}\text { Iraq: Refugee } \\
\text { camps/informal } \\
\text { settlements }\end{array}$ & Refugees only & $\begin{array}{l}\text { Urban agriculture, } \\
\text { animals, foraging }\end{array}$ & No & No \\
\hline Millican J, 2019 [63] & $\begin{array}{l}\text { Iraq: Refugee } \\
\text { camps/informal } \\
\text { settlements }\end{array}$ & Refugees only & $\begin{array}{l}\text { Urban agriculture, } \\
\text { animals, foraging }\end{array}$ & No & No \\
\hline Giordano N, 2017 [50] & $\begin{array}{l}\text { Jordan: Outside } \\
\text { camps }\end{array}$ & Refugees only & Cash & No & $\begin{array}{l}\text { Questions on \# of } \\
\text { meals, diet } \\
\text { diversity, food } \\
\text { frequency, coping } \\
\text { strategies. }\end{array}$ \\
\hline Fander G, 2014 [44] & $\begin{array}{l}\text { Jordan: Inside and } \\
\text { outside camps }\end{array}$ & $\begin{array}{l}\text { Refugees and host } \\
\text { communities }\end{array}$ & $\begin{array}{l}\text { IYCF * and } \\
\text { pregnancy }\end{array}$ & Yes & No \\
\hline Alsamman S, 2014 [34] & $\begin{array}{l}\text { Jordan: Refugee } \\
\text { camps/informal } \\
\text { settlements }\end{array}$ & Refugees only & $\begin{array}{l}\mathrm{IYCF}^{*} \text { and } \\
\text { pregnancy }\end{array}$ & Yes & No \\
\hline Sebuliba H, 2014 [69] & $\begin{array}{l}\text { Jordan: Inside and } \\
\text { outside camps }\end{array}$ & Refugees only & $\begin{array}{l}\text { IYCF * and } \\
\text { pregnancy }\end{array}$ & Yes & No \\
\hline $\begin{array}{l}\text { Boston Consulting } \\
\text { Group, } 2017 \text { [39] }\end{array}$ & $\begin{array}{c}\text { Jordan and } \\
\text { Lebanon: Outside } \\
\text { camps }\end{array}$ & Refugees only & $\begin{array}{l}\text { Mixed cash, } \\
\text { vouchers, food } \\
\text { transfers }\end{array}$ & No & $\begin{array}{l}\text { Consolidated } \\
\text { Approach for } \\
\text { Reporting } \\
\text { Indicators }\end{array}$ \\
\hline $\begin{array}{l}\text { Abu Hamad B, } \\
2017 \text { [32] }\end{array}$ & $\begin{array}{l}\text { Jordan: Outside } \\
\text { camps }\end{array}$ & Refugees only & $\begin{array}{l}\text { Mixed cash, } \\
\text { vouchers, food } \\
\text { transfers }\end{array}$ & No & $\begin{array}{c}\text { Food } \\
\text { Consumption } \\
\text { Score }\end{array}$ \\
\hline $\begin{array}{c}\text { World Food } \\
\text { Programme, 2015a [5] }\end{array}$ & $\begin{array}{l}\text { Jordan: Inside and } \\
\text { outside camps }\end{array}$ & Refugees only & $\begin{array}{l}\text { Mixed cash, } \\
\text { vouchers, food } \\
\text { transfers }\end{array}$ & No & $\begin{array}{c}\text { Food } \\
\text { Consumption } \\
\text { Scores and the } \\
\text { Coping Strategy } \\
\text { Index }\end{array}$ \\
\hline $\begin{array}{c}\text { World Food } \\
\text { Programme, 2018a [83] }\end{array}$ & $\begin{array}{l}\text { Kenya: Refugee } \\
\text { camps/informal } \\
\text { settlements }\end{array}$ & Refugees only & Cash & No & $\begin{array}{c}\text { Household Dietary } \\
\text { Diversity Score, } \\
\text { Food } \\
\text { Consumption } \\
\text { Score, Coping } \\
\text { Strategies Index }\end{array}$ \\
\hline Betts A, 2020 [37] & $\begin{array}{l}\text { Kenya: Inside and } \\
\text { outside camps }\end{array}$ & Refugees only & $\begin{array}{l}\text { Mixed cash, } \\
\text { vouchers, food } \\
\text { transfers }\end{array}$ & No & $\begin{array}{c}\text { Household Food } \\
\text { Insecurity Access } \\
\text { Prevalence; } \\
\text { Dietary Diversity } \\
\text { Score; Food } \\
\text { Consumption } \\
\text { Score }\end{array}$ \\
\hline
\end{tabular}


Table 2. Cont.

\begin{tabular}{|c|c|c|c|c|c|}
\hline First Author, Year & Location & Target Population & Intervention Type & $\begin{array}{c}\text { Considers } \\
\text { Gender }\end{array}$ & $\begin{array}{c}\text { Food Security } \\
\text { Measure }\end{array}$ \\
\hline Oka R, 2011 [66] & $\begin{array}{l}\text { Kenya: Refugee } \\
\text { camps/informal } \\
\text { settlements }\end{array}$ & Refugees only & $\begin{array}{c}\text { Informal } \\
\text { economy/trading }\end{array}$ & No & No \\
\hline Battistin F, 2018 [36] & $\begin{array}{c}\text { Lebanon: Outside } \\
\text { camps }\end{array}$ & Refugees only & Cash & No & $\begin{array}{c}\text { Food } \\
\text { Consumption } \\
\text { Score, Household } \\
\text { Weekly Dietary } \\
\text { Diversity Score, } \\
\text { Household Daily } \\
\text { Average Dietary } \\
\text { Diversity Score }\end{array}$ \\
\hline Ibrahim, N., 2019 [58] & $\begin{array}{c}\text { Lebanon: Refugee } \\
\text { camps/informal } \\
\text { settlements }\end{array}$ & $\begin{array}{l}\text { Refugees and host } \\
\text { communities }\end{array}$ & $\begin{array}{l}\text { Community } \\
\text { kitchen }\end{array}$ & Yes & $\begin{array}{l}\text { Questions on } \\
\text { types, amount, and } \\
\text { variety of food, } \\
\text { nutritional value, } \\
\text { for chronic } \\
\text { conditions, } \\
\text { preference, culture, } \\
\text { finances, what } \\
\text { happens when } \\
\text { food runs out, } \\
\text { supplemental } \\
\text { food. }\end{array}$ \\
\hline Ghattas H, 2019 [48] & $\begin{array}{l}\text { Lebanon: Refugee } \\
\text { camps/informal } \\
\text { settlements }\end{array}$ & Refugees only & $\begin{array}{c}\text { Community } \\
\text { kitchen/School- } \\
\text { based } \\
\text { nutrition }\end{array}$ & Yes & $\begin{array}{l}\text { Arab Family Food } \\
\text { Security Scale }\end{array}$ \\
\hline Aste N, 2017 [35] & $\begin{array}{c}\text { Lebanon: Outside } \\
\text { camps }\end{array}$ & Refugees only & $\begin{array}{c}\text { Food safety and } \\
\text { energy }\end{array}$ & No & No \\
\hline $\begin{array}{l}\text { El Harake MD, } \\
2018 \text { [43] }\end{array}$ & $\begin{array}{l}\text { Lebanon: Refugee } \\
\text { camps/informal } \\
\text { settlements }\end{array}$ & Refugees only & $\begin{array}{l}\text { School-based } \\
\text { nutrition }\end{array}$ & Yes & $\begin{array}{l}\text { Arabic-translated, } \\
\text { locally validated } \\
\text { version of the } \\
\text { Household Food } \\
\text { Insecurity Access } \\
\text { Scale }\end{array}$ \\
\hline Dehnavi S, 2019 [41] & $\begin{array}{c}\text { Lebanon: Outside } \\
\text { camps }\end{array}$ & $\begin{array}{l}\text { Refugees and host } \\
\text { communities }\end{array}$ & $\begin{array}{l}\text { Urban agriculture, } \\
\text { animals, foraging }\end{array}$ & No & No \\
\hline $\begin{array}{l}\text { Food and Agriculture } \\
\text { Organization of the } \\
\text { United Nations, } \\
2016 \text { [45] }\end{array}$ & $\begin{array}{l}\text { Lebanon: Inside } \\
\text { and outside camps }\end{array}$ & $\begin{array}{l}\text { Refugees and host } \\
\text { communities }\end{array}$ & $\begin{array}{l}\text { Urban agriculture, } \\
\text { animals, foraging }\end{array}$ & Yes & No \\
\hline Sub V, 2018 [72] & $\begin{array}{c}\text { Lebanon: Outside } \\
\text { camps }\end{array}$ & $\begin{array}{l}\text { Refugees and host } \\
\text { communities }\end{array}$ & $\begin{array}{l}\text { Urban agriculture, } \\
\text { animals, foraging }\end{array}$ & No & $\begin{array}{c}\text { 6-item short form } \\
\text { of 18-item Food } \\
\text { Security } \\
\text { Measurement } \\
\text { Module by the } \\
\text { United States } \\
\text { Department of } \\
\text { Agriculture }\end{array}$ \\
\hline
\end{tabular}


Table 2. Cont.

\begin{tabular}{|c|c|c|c|c|c|}
\hline First Author, Year & Location & Target Population & Intervention Type & $\begin{array}{l}\text { Considers } \\
\text { Gender }\end{array}$ & $\begin{array}{l}\text { Food Security } \\
\text { Measure }\end{array}$ \\
\hline $\begin{array}{c}\text { World Food } \\
\text { Programme, 2016b [81] }\end{array}$ & $\begin{array}{l}\text { Liberia: Inside and } \\
\text { outside camps }\end{array}$ & $\begin{array}{l}\text { Refugees and host } \\
\text { communities }\end{array}$ & $\begin{array}{l}\text { Mixed cash, } \\
\text { vouchers, food } \\
\text { transfers }\end{array}$ & No & $\begin{array}{c}\text { Food } \\
\text { Consumption } \\
\text { Score }\end{array}$ \\
\hline $\begin{array}{c}\text { World Food } \\
\text { Programme, } 2014 \text { [78] }\end{array}$ & $\begin{array}{c}\text { Mozambique: } \\
\text { Inside and outside } \\
\text { camps }\end{array}$ & $\begin{array}{l}\text { Refugees and host } \\
\text { communities }\end{array}$ & $\begin{array}{l}\text { Mixed cash, } \\
\text { vouchers, food } \\
\text { transfers }\end{array}$ & No & $\begin{array}{l}\text { Emergency Food } \\
\text { Security } \\
\text { Assessment, } \\
\text { Comprehensive } \\
\text { Food Security and } \\
\text { Vulnerability } \\
\text { Analysis }\end{array}$ \\
\hline Qleibo E, 2013 [68] & $\begin{array}{l}\text { Palestine (Gaza): } \\
\text { Outside camps }\end{array}$ & $\begin{array}{l}\text { Refugees and host } \\
\text { communities }\end{array}$ & $\begin{array}{l}\text { Urban agriculture, } \\
\text { animals, foraging }\end{array}$ & Yes & No \\
\hline $\begin{array}{c}\text { Karama Organization, } \\
2015[60]\end{array}$ & $\begin{array}{l}\text { Palestine: Refugee } \\
\text { camps/informal } \\
\text { settlements }\end{array}$ & Refugees only & $\begin{array}{l}\text { Urban agriculture, } \\
\text { animals, foraging }\end{array}$ & Yes & No \\
\hline Alloush M, 2017 [33] & $\begin{array}{l}\text { Rwanda: Refugee } \\
\text { camps/informal } \\
\text { settlements }\end{array}$ & Refugees only & $\begin{array}{l}\text { Mixed cash, } \\
\text { vouchers, food } \\
\text { transfers }\end{array}$ & No & $\begin{array}{l}\text { One question: "In } \\
\text { last } 7 \text { days, have } \\
\text { there been times } \\
\text { when household } \\
\text { did not have } \\
\text { enough or money } \\
\text { to buy food?" }\end{array}$ \\
\hline $\begin{array}{c}\text { World Food } \\
\text { Programme, 2016c [82] }\end{array}$ & $\begin{array}{l}\text { Rwanda: Inside } \\
\text { and outside camps }\end{array}$ & $\begin{array}{l}\text { Refugees and host } \\
\text { communities }\end{array}$ & $\begin{array}{l}\text { Mixed cash, } \\
\text { vouchers, food } \\
\text { transfers }\end{array}$ & Yes & $\begin{array}{c}\text { Food } \\
\text { Consumption } \\
\text { Score }\end{array}$ \\
\hline de Bruin N, 2019 [40] & $\begin{array}{c}\text { Tanzania: Refugee } \\
\text { camps/informal } \\
\text { settlements }\end{array}$ & $\begin{array}{l}\text { Refugees and host } \\
\text { communities }\end{array}$ & $\begin{array}{l}\text { Mixed cash, } \\
\text { vouchers, food } \\
\text { transfers }\end{array}$ & No & No \\
\hline Hashmi A, 2019 [55] & $\begin{array}{c}\text { Thailand: Refugee } \\
\text { camps/informal } \\
\text { settlements }\end{array}$ & Refugees only & $\begin{array}{l}\text { IYCF* and } \\
\text { pregnancy }\end{array}$ & Yes & No \\
\hline Stuetz W, 2016 [71] & $\begin{array}{l}\text { Thailand: Refugee } \\
\text { camps/informal } \\
\text { settlements }\end{array}$ & Refugees only & $\begin{array}{l}\mathrm{IYCF} * \text { and } \\
\text { pregnancy }\end{array}$ & Yes & No \\
\hline Inglis K, 2014 [59] & $\begin{array}{l}\text { Turkey: Refugee } \\
\text { camps/informal } \\
\text { settlements }\end{array}$ & Refugees only & Voucher & No & No \\
\hline Mochizuki Y, 2017 [64] & $\begin{array}{l}\text { Uganda: Refugee } \\
\text { camps/informal } \\
\text { settlements }\end{array}$ & Refugees only & $\begin{array}{l}\text { Urban agriculture, } \\
\text { animals, foraging }\end{array}$ & No & No \\
\hline $\begin{array}{c}\text { Food and Agriculture } \\
\text { Organization of the } \\
\text { United Nations, } \\
2018 \text { [46] }\end{array}$ & $\begin{array}{l}\text { Uganda: Outside } \\
\text { camps }\end{array}$ & $\begin{array}{l}\text { Refugees and host } \\
\text { communities }\end{array}$ & $\begin{array}{l}\text { Urban agriculture, } \\
\text { animals, foraging }\end{array}$ & Yes & No \\
\hline Smock L, 2020 [70] & $\begin{array}{l}\text { USA: Destination } \\
\text { country }\end{array}$ & Refugees only & $\begin{array}{l}\text { IYCF * and } \\
\text { pregnancy }\end{array}$ & Yes & No \\
\hline Trapp M, 2010 [75] & $\begin{array}{l}\text { USA: Destination } \\
\text { country }\end{array}$ & Refugees only & $\begin{array}{c}\text { Nutrition } \\
\text { education } \\
\text { programming }\end{array}$ & Yes & No \\
\hline
\end{tabular}


Table 2. Cont.

\begin{tabular}{|c|c|c|c|c|c|}
\hline First Author, Year & Location & Target Population & Intervention Type & $\begin{array}{l}\text { Considers } \\
\text { Gender }\end{array}$ & $\begin{array}{l}\text { Food Security } \\
\text { Measure }\end{array}$ \\
\hline Bloom JD, 2018 [38] & $\begin{array}{l}\text { USA: Destination } \\
\text { country }\end{array}$ & $\begin{array}{l}\text { Refugees and host } \\
\text { communities }\end{array}$ & $\begin{array}{c}\text { Nutrition } \\
\text { education } \\
\text { programming }\end{array}$ & Yes & No \\
\hline Gold A, 2014 [52] & $\begin{array}{l}\text { USA: Destination } \\
\text { country }\end{array}$ & $\begin{array}{l}\text { Refugees and host } \\
\text { communities }\end{array}$ & $\begin{array}{c}\text { Nutrition } \\
\text { education } \\
\text { programming }\end{array}$ & No & No \\
\hline Gunnell S, 2015 [53] & $\begin{array}{l}\text { USA: Destination } \\
\text { country }\end{array}$ & Refugees only & $\begin{array}{c}\text { Nutrition } \\
\text { education } \\
\text { programming }\end{array}$ & No & No \\
\hline McElrone M, 2020 [62] & $\begin{array}{l}\text { USA: Destination } \\
\text { country }\end{array}$ & Refugees only & $\begin{array}{c}\text { Nutrition } \\
\text { education } \\
\text { programming }\end{array}$ & Yes & $\begin{array}{l}\text { Measured, tool not } \\
\text { specified. Food } \\
\text { Security Score: } \\
26 \text { items for adults- } \\
\text { cooking, eating, } \\
\text { playing together, } \\
\text { kitchen proficiency, } \\
\text { and food security. }\end{array}$ \\
\hline Eggert LK, 2015 [42] & $\begin{array}{l}\text { USA: Destination } \\
\text { country }\end{array}$ & Refugees only & $\begin{array}{l}\text { Urban agriculture, } \\
\text { animals, foraging }\end{array}$ & No & No \\
\hline Hartwig KA, 2016 [54] & $\begin{array}{l}\text { USA: Destination } \\
\text { country }\end{array}$ & $\begin{array}{l}\text { Refugees and host } \\
\text { communities }\end{array}$ & $\begin{array}{l}\text { Urban agriculture, } \\
\text { animals, foraging }\end{array}$ & No & $\begin{array}{c}\text { Hunger assessed } \\
\text { using } \\
\text { internationally } \\
\text { validated food } \\
\text { security questions } \\
\text { by the UN Food } \\
\text { and Agriculture } \\
\text { Organization }\end{array}$ \\
\hline Volpato G, 2013 [75] & $\begin{array}{l}\text { Western Sahara, } \\
\text { Mauritania, } \\
\text { Algeria: Inside and } \\
\text { outside camps }\end{array}$ & Refugees only & $\begin{array}{l}\text { Urban agriculture, } \\
\text { animals, foraging }\end{array}$ & No & No \\
\hline Volpato G, 2014 [76] & $\begin{array}{c}\text { Western Sahara: } \\
\text { Inside and outside } \\
\text { camps }\end{array}$ & Refugees only & $\begin{array}{l}\text { Urban agriculture, } \\
\text { animals, foraging }\end{array}$ & No & No \\
\hline
\end{tabular}

${ }^{*}$ IYCF $=$ Infant and Young Child Feeding. 


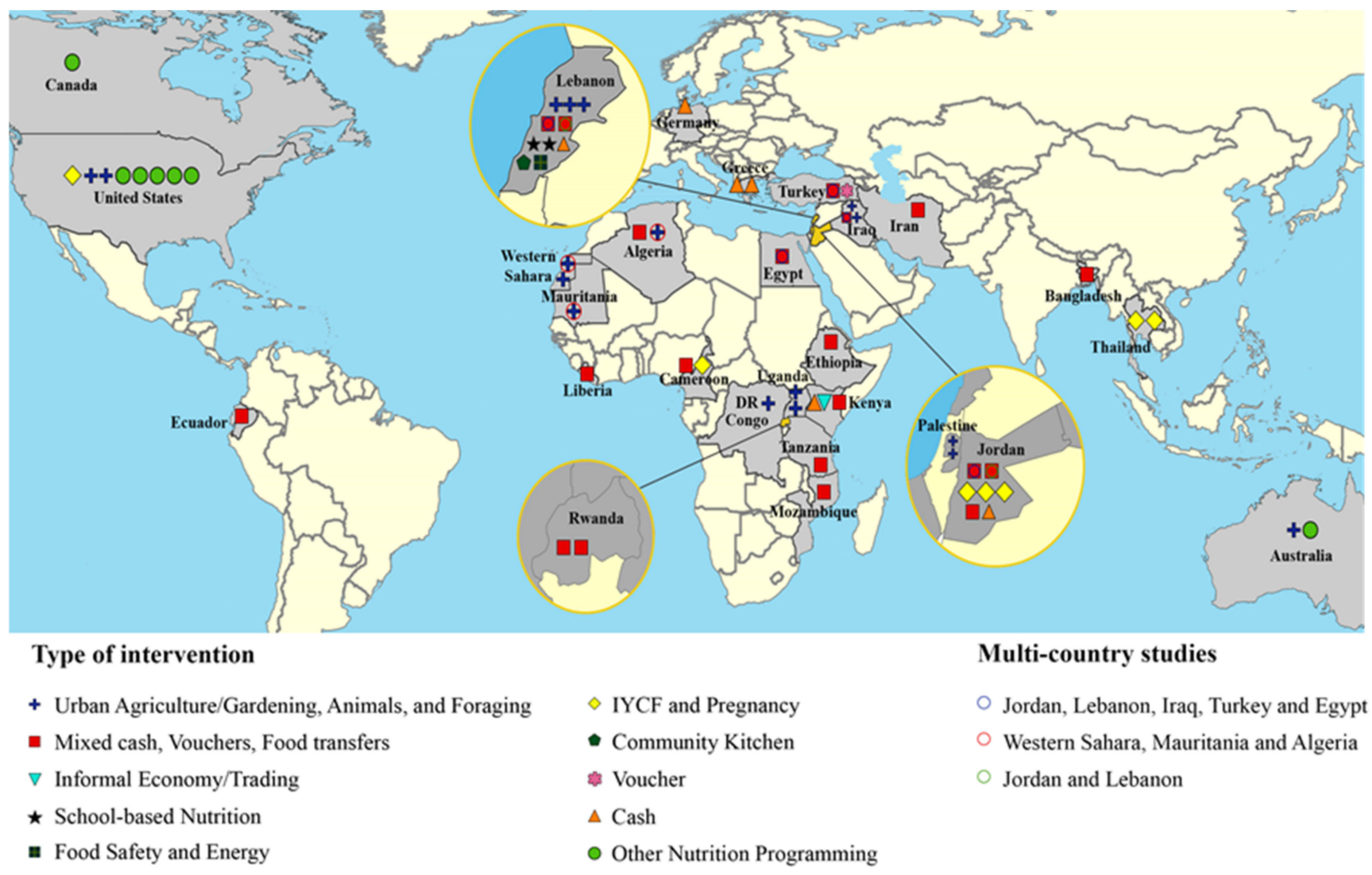

Figure 4. Intervention types according to country.

Food security was directly measured in 39\% of studies. While the remainder addressed food security with interventions such as urban agriculture, infant and young child feeding, and nutrition education programs, they did not include direct assessment. While $52 \%$ of studies that measured food security used the United Nations Food Consumption Score alone or in addition to the accompanying Diet Diversity Score or Coping Strategies Index, the other $48 \%$ each used different methods to measure food security. These methods used one question or multiple questions to assess food security status. Although many seem to be based on the FAO, the WFP, or the United States Department of Agriculture (USDA) methods - the three most well-known validated questionnaires in developing and developed countries-there is no indication that they were validated questionnaires.

\section{Discussion}

In this review, we evaluated food security interventions in refugees and existing gaps in knowledge. Overall, 57 studies met the inclusion criteria, mainly in the area of refugee crisis. Consistently high levels of food insecurity among refugees indicate a need for one standard tool to measure food security across locations to improve understanding around food security in different contexts and help determine best practices and policies. This review has discovered multiple gaps in research leading to limited knowledge of the efficacy of interventions in different refugee settings.

\subsection{Summary of Evidence}

4.1.1. Intervention Types across Geographic Locations

Areas of Refugee Crisis

Most studies in areas of refugee crisis such as the Middle East and Southeast Africa report on interventions that include a mixture of cash, vouchers, and food transfers (Figure 3). Substantive literature exists on types of interventions, providing evidence for cash-based transfers as opposed to vouchers or food rations as cash provides choice, flexibility, sense of dignity, and empowerment $[23,32,33]$. However, in areas where markets are not developed, 
such as newly established refugee camps, rations seem to be the most beneficial until informal and/or formal economies are established, and markets stabilize. When providing assistance, it is important to consider gender, the inclusion of host communities in the interventions, and the accompaniment of livelihood strategies.

When examining intervention types by UN agencies, we observed that urban agriculture was a focus of the FAO, and cash/voucher interventions were implemented by the WFP; however, there was not much mention of these two agencies working together to combine efforts. The FAO aims to achieve food security for all, the mandate of the UNHCR is to provide international protection to refugees and other persons of concern, and the role of the WFP is to use food aid to support economic and social development, meet food needs in emergency and protracted situations, and promote food security based on FAO recommendations [87,88]. Despite documents such as the 2011 Memorandum of Understanding between UNHCR and WFP being in place, details of these collaborations are lacking, and evaluations of UN agency programs recommend collaboration [81,88]. For example, a 2016 evaluation of WFP programs in Liberia indicated that UNICEF and FAO are listed as partners in the project document, yet no evidence of this collaboration could be found by the evaluation team in any other documentation. Inter-agency action-oriented collaboration could maximize resources, streamline services, and allow the development of successful plans for a transition from cash assistance to livelihood strategies and thus programmatic sustainability.

Based on recommendations from UN agency impact evaluations, in July 2020, the UNHCR and the WFP announced the launch of the "Joint Strategy for Enhancing SelfReliance in Food Security and Nutrition in Protracted Refugee Situations" [89]. They will assess the refugee situation together, investigate the vulnerabilities, capacities and opportunities together based on their assessment, and set goals to improve self-reliance and livelihoods [89]. They will also evaluate their progress on self-reliance in food security together [89]. The new strategy has two main objectives that focus on empowering refugees and creating a supportive environment by engaging the local government and host communities [89]. Although the new joint strategy seems promising and focuses on empowering refugees by engaging all stakeholders, to our knowledge, there is no evidence to evaluate its effectiveness.

In areas of refugee crisis, when host communities are not involved in interventions, it creates feelings of hostility towards refugees as host communities feel like refugees are being helped above their own most vulnerable. The refugee-host relationship can also be affected by country policies which limit the rights of refugees limiting freedom of movement, access to work visas, ownership of land, and more, which is beyond the scope of this review. Including host communities when targeting households for food assistance improves the refugee-host relationship [46].

Livelihood strategies are important to improve sustainability of the aid provided and assist refugees in becoming self-sufficient, particularly when aid is often reduced [11]. It is of note to mention that not all interventions are purposeful, and some are instigated by refugees themselves in the form of establishing informal economies and trading in and around refugee camps [90]. It is beneficial to take note of these interventions as well because we can learn from the entrepreneurial activities of refugees when planning interventions as it is indicative of what refugees need. By providing more livelihood opportunities with the support of humanitarian aid agencies, it may be possible to improve refugee self-reliance, empowerment, and gender equity $[46,47,60,76]$.

A considerable amount of evidence is focused on Palestinian and Syrian refugees in Lebanon and Jordan $[5,41,43,48,58]$. While most interventions in refugee crisis areas are focused on cash, vouchers, and food transfers, studies in Lebanon reported more sustainable programs such as school-based nutrition, community kitchens and urban agriculture, which are in the line of main interventions in developed destination countries $[41,43,45,48,58]$. 


\section{Destination Countries}

Refugees are a vulnerable population that suffer unique challenges that often affect their food security status even after entering destination countries. Our results showed few studies are being conducted on refugee food security interventions in developed destination countries despite similar levels of food insecurity between refugees in destination and nondestination countries [6,91]. For example, a Canadian study by Lane et al. (2019) reported that $50 \%$ of refugee households (from various countries of origin) were food insecure [91]. Similarly, 50\% of Syrian refugees in Lebanon have been found to be food insecure [6]. It is also common to see studies in destination countries (e.g., Canadian Community Health Survey in Canada) grouping refugees with immigrants and excluding participants who cannot speak the country's official languages, which portrays an inaccurate and underestimated image of refugee food security issues [92]. Only 17\% of refugee food security intervention studies in destination countries measured food security status.

There is a significant difference in the types of interventions in developed destination countries focusing mainly on urban agriculture, gardening, animal husbandry, and foraging, and other nutrition programming such as nutrition education (Figure 3 ). In destination countries such as Canada, refugees are covered by direct cash support and housing programs in the first year of arrival [93]. Afterwards, based on their situation, they could be eligible for regular social assistance programs. An abrupt cessation to federal government aid may explain the high prevalence of food insecurity among refugees in destination countries a year after arrival [27].

\subsubsection{Considerations for the Most Vulnerable}

Gender is an important consideration when developing food security interventions. In many cultures, women are often in charge of food preparation for the household. We know that women/mothers are more likely to cut back their intake and portion sizes so that other families, particularly children, can have enough to eat [17]. Women are more likely to be food insecure and women and girls are at greater risk of gender-based violence $[50,82,85,94]$. Although many UN agency interventions included gender considerations in the intervention plan (e.g., planned to target women as beneficiaries of cash/food transfers), evaluations showed that these considerations are lacking during implementation $[81,85]$. Evaluations often indicated a need for more security, oversight, monitoring, and evaluation in camp settings $[81,84]$. Equitable gender considerations can be difficult because many countries still lack women's rights and their policies and social norms may prevent women from seeking employment outside the home, and other genders are not considered due to discrimination and oppressive laws [14,32,94]. Few studies mentioned other at-risk populations such as children not covered by IYCF programs, the elderly, LGBTQIA2S+, and persons with disabilities, and research shows that these people are often overlooked in the design and implementation of humanitarian aid, indicating a need to amend interventions to assist these at-risk groups [14,15]. Although not all interventions can affect policy change, it is important to work with governments to find ways to assist the most vulnerable.

\subsubsection{Assessing Food Security}

Our review showed that less than half of the studies that aimed to address food security issues actually measured food security, and those that did used a variety of different tools with only some being validated. The most common tool used to measure food security was the UN's Food Consumption Score used in 52\% of the studies that measured food security, while all other tools were only used in one study each. A wide range of food security topics makes it difficult to assess the efficacy of interventions. A consistent tool that is validated in different languages is needed to accurately compare food security across locations and contexts, differentiating between adult and child food security and providing a more complete picture of food security issues in households, which would allow more targeted interventions. The WFP is evaluating the food security status of refugees in areas 
of refugee crises using the Consolidated Approach to Reporting Indicators of Food Security (CARI) [95]. This comprehensive tool incorporates the Food Consumption Score, economic capacity, and livelihood coping strategies, which has been widely accepted and is a good measure of food security [95]. The Household Food Security Survey Module (HFSSM) is a questionnaire containing 18 questions that assess income-related food security status at household, adult, and child levels [96]. The HFSSM has been validated and used in more than 19 languages in different countries, particularly developed destination countries [96]. The ability of the HFSSM to assess food security in households, adults, and children makes it a proper candidate as a standard tool that fills the gap in our ability to universally assess the efficacy of food security interventions in different settings. Destination countries such as Canada and the USA are using the HFSSM regularly in their nutrition and health national surveys [96]. Therefore, using either tool or a combination as a standard food security assessment tool will allow the comparison of food security status of refugees with host countries to identify the gaps and disparities.

\subsection{Knowledge Gaps and Research Recommendations}

A considerable number of studies in areas of refugee crisis evaluated the short-term interventions of international agencies individually [23,81,82]. There is a lack of evidence as to whether international agencies are working together on interventions they support collectively and, if so, how effective those initiatives are compared to interventions implemented by one agency alone. Further, it is not clear the extent to which international agencies work with local governments or NGOs on the sustainability of interventions that is necessary to empower refugees, enable them to be self-sufficient, improve their food security status, and contribute to local economies.

Research has shown that beneficiaries prefer cash to vouchers and rations and that cash often results in better outcomes compared to other modalities $[32,33,39,40,56]$. The lack of direct food security measures in many studies, along with insufficient methodologies (e.g., measures only in one time point, lack of food security measures, lack of control group), prevented an assessment of any improvement correlated with the intervention itself. The lack of a consistent tool used to measure food security prevents any comparison across studies, which goes beyond the scope of this review. Similarly, limited studies on cash, vouchers, and/or rations measured food security and considered gender in their implementation. Of those that did, none compared food security results across genders.

Grey literature indicates the role of community-based organizations and host communities in supporting and empowering refugees, particularly in destination countries [60,61,77]. Such organizations conduct interventions without proper pre- and post-evaluations, leading to lack of evidence on the impact and effectiveness of such initiatives. There is a need to identify, evaluate, and document best practices aimed to improve the food security status of refugees.

Although international agencies have clear policies and work plans with regards to food security in areas of refugee crisis, to our knowledge no study has evaluated the policies by local governments in areas of refugee crisis as well as destination countries $[88,97,98]$. Such studies will assist in identifying effective policies that aim to improve food security status of refugees while empowering them as new members of the host community.

Short-term interventions are necessary to alleviate hunger and other short-term effects of food insecurity among refugees. However, many protracted refugees continue to live in unstable situations in host countries, which can impact their food security status. There is limited information surrounding food security interventions in protracted crises, likely due to limited resources and international aid agencies focusing efforts on acute crises. Thus, further efforts are required to address sustainability issues when it comes to food security interventions. 


\subsection{Strengths and Limitations}

To our knowledge, this is the first study to use a systematic approach using the PRISMA guidelines to identify and evaluate the selected literature on refugee food security interventions. The main strength of our study is the systematic method of setting eligibility criteria, identifying the literature, and detecting the gaps in research. The categorization of types of interventions and geo-mapping according to geographic locations is another strength of our study that provides insight into the distribution of the types of interventions across the globe.

Regarding limitations, we only included interventions reported in the English language as indicated in the inclusion criteria. Therefore, we were unable to identify and include reports available in different local languages. The variation in the tools used to assess food security and methods of evaluation limited us from having an overall picture of food insecurity status before the evaluation of the effectiveness of interventions.

\section{Conclusions}

Refugee crisis is on the rise due to climate change, war and other political and societal conflicts. Humanitarian agencies continually provide assistance and evaluate their interventions in areas of refugee crisis. The resultant evidence has provided substantive information on when to use certain types of interventions, such as cash when markets are stable and the importance of incorporating livelihood strategies to transition to a sustainable level of aid and help refugees become self-sufficient and active members of their communities. In destination countries, the types of interventions are more towards capacity building and education. Considering numerous existing interventions, the rate of food insecurity is still very high among refugees. In addition, due to lack of a proper and universal approach for evaluation, the efficacy of interventions is not clear. Further efforts are necessary to work with governments to affect policy change to advocate for the rights of marginalized populations such as children, seniors, women, LGBTQIA2S+, persons with disabilities, and minority groups. It is also vital to engage host communities and NGOs to create a welcoming culture that benefits both refugees and host communities. Finally, researchers should adopt a standard feasible food security assessment tool which is needed to assess the effectiveness of interventions across locations and countries to develop best practices based on comparative results.

Supplementary Materials: The following supporting information can be downloaded at: https: / / www.mdpi.com/article/10.3390/nu14030522/s1; Table S1: Sample search strategy.

Author Contributions: Conceptualization, C.N. and H.V.; methodology, C.N. and H.V.; formal analysis, C.N. and K.E.L.; data curation, C.N. and K.E.L.; writing - original draft preparation, C.N. and H.V.; writing-review and editing, C.N. and H.V.; visualization, C.N. and H.V.; supervision, H.V.; project administration, C.N.; funding acquisition, H.V. All authors have read and agreed to the published version of the manuscript.

Funding: This research was funded by The Social Sciences and Humanities Research Council (SSHRC) Canada, Fund number 423118.

Institutional Review Board Statement: Not applicable.

Informed Consent Statement: Not applicable.

Data Availability Statement: Data sharing not applicable to this scoping review.

Conflicts of Interest: The authors declare no conflict of interest. 


\section{References}

1. The UN Refugee Agency. Convention and Protocol Relating to the Status of Refugees; The UN Refugee Agency: Geneva, Switzerland, 2010. Available online: https://www.unhcr.org/protection/basic/3b66c2aa10/convention-protocol-relating-status-refugees. html (accessed on 1 August 2021).

2. The UN Refugee Agency. Internally Displaced People; The UN Refugee Agency: Geneva, Switzerland, 2021. Available online: https:/ / www.unhcr.org/internally-displaced-people.html (accessed on 1 August 2021).

3. The UN Refugee Agency. Asylum-Seekers; The UN Refugee Agency: Geneva, Switzerland, 2021. Available online: https: //www.unhcr.org/asylum-seekers.html (accessed on 1 August 2021).

4. The UN Refugee Agency. Figures at a Glance; The UN Refugee Agency: Geneva, Switzerland, 2020. Available online: https: //www.unhcr.org/figures-at-a-glance.html (accessed on 1 August 2021).

5. World Food Programme. An Evaluation of WFP's Regional Response to the Syrian Crisis 2011-2014; World Food Programme: Rome, Italy, 2015. Available online: https:/ / www.wfp.org/publications/evaluation-wfps-regional-response-syrian-crisis-2011-2014 (accessed on 28 November 2020).

6. The UN Refugee Agency. Regional Refugee and Resilience Plan (3RP): In Response to the Syria Crisis: Regional Strategic Overview 2021-2022; The UN Refugee Agency: Geneva, Switzerland, 2020. Available online: http://www.3rpsyriacrisis.org/wp-content/ uploads/2021/03/RSO_2March2021upd.pdf (accessed on 23 November 2020).

7. The UN Refugee Agency. Operational Portal Refugee Situations: Mediterranean Situation; The UN Refugee Agency: Geneva, Switzerland, 2021. Available online: http:/ / data2.unhcr.org/en/situations/mediterranean-_ga=2.231906713.787304818.159871 9474-1502710622.1598291859 (accessed on 15 October 2020).

8. The UN Refugee Agency. Global Trends: Forced Displacement in 2019; The UN Refugee Agency: Geneva, Switzerland, 2020. Available online: https:/ / www.unhcr.org/5ee200e37.pdf (accessed on 25 November 2020).

9. Food and Agriculture Organization of the United Nations. World Food Summit: Rome Declaration on World Food Security; Food and Agriculture Organization of the United Nations: Rome, Italy, 1996. Available online: http://www.fao.org/3/w3613e/w3613e00. htm (accessed on 3 July 2021).

10. Committee on World Food Security. Global Strategic Framework for Food Security and Nutrition; Committee on World Food Security: Rome, Italy, 2014. Available online: http:/ / www.fao.org/cfs/policy-products/gsf/en/ (accessed on 15 September 2020).

11. Oliver, M.; Ilcan, S. The politics of protection: The right to food in protracted refugee situations. Refug. Surv. Q. 2018, 37, 440-457. [CrossRef]

12. U.S Department of State; Bureau of Population, Refugees, and Migration. At-Risk Populations. Available online: https: / / www.state.gov/other-policy-issues/at-risk-populations/ (accessed on 27 December 2021).

13. Women at Risk International. Refugees at Risk. Available online: https://warinternational.org/refugees-at-risk/ (accessed on 27 December 2021).

14. World Economic Forum. Forgotten Twice: The Untold Story of LGBT Refugees; World Economic Forum: Cologny, Switzerland, 2018. Available online: https://www.weforum.org/agenda/2018/01/forgotten-twice-lgbt-refugees/ (accessed on 27 December 2021)

15. Burton, A.; Breen, C. Older refugees in humanitarian emergencies. Lancet Suppl. 2002, 360, s47-s48. [CrossRef]

16. Food and Agriculture Organization of the United Nations; International Fund for Agricultural Development; United Nations Children's Fund; World Food Programme; World Health Organization. The State of Food Security and Nutrition in the World 2020: Safeguarding against Economic Slowdowns and Downturns; Food and Agriculture Organization of the United Nations: Rome, Italy, 2020. [CrossRef]

17. Matheson, J.; McIntyre, L. Women respondents report higher household food insecurity than do men in similar Canadian households. Public Health Nutr. 2014, 17, 40-48. [CrossRef] [PubMed]

18. Haddad, L.J.; Peña, C.; Nishida, C.; Quisumbing, A.R.; Slack, A. Food Security and Nutrition Implications of Intrahousehold Bias: A Review of Literature; Food Consumption and Nutrition Division, International Food Policy Research Institute: Washington, DC, USA, 1996. Available online: https:/ / www.ifpri.org/publication/food-security-and-nutrition-implications-intrahousehold-bias (accessed on 15 September 2020).

19. Moffat, T.; Mohammed, C.; Newbold, K.B. Cultural dimensions of food insecurity among immigrants and refugees. Hum. Organ. 2017, 76, 15-27. [CrossRef]

20. Development Initiatives Poverty Research Ltd. 2020 Global Nutrition Report; Development Initiatives Poverty Research Ltd.: Bristol, UK, 2020. Available online: https://globalnutritionreport.org/reports/2020-global-nutrition-report/ (accessed on 15 September 2020).

21. The UN Refugee Agency. Nutrition and Food Security; The UN Refugee Agency: Geneva, Switzerland, 2021. Available online: https:/ / www.unhcr.org/nutrition-and-food-security.html (accessed on 10 October 2020).

22. International Disability Alliance. Disability and Food Security: An Unfinished Policy Agenda; International Disability Alliance: New York, NY, USA, 2018. Available online: https:/ / www.internationaldisabilityalliance.org/fao-food-insecurity (accessed on 27 December 2021).

23. Dunlop, K.; Ellina, M.; Smith, E. Evaluation of the Effects of Cash Based Interventions on Protection Outcomes in Greece. In Action Against Hunger UK's Monitoring, Evaluation and Learning Services; The UN Refugee Agency: Geneva, Switzerland, 2018.

24. Carroll, G.J.; Lama, S.D.; Martinez-Brockman, J.L.; Pérez-Escamilla, R. Evaluation of nutrition interventions in children in conflict zones: A narrative review. Adv. Nutr. 2017, 8, 770-779. [CrossRef] [PubMed] 
25. The UN Refugee Agency. Ending Statelessness; The UN Refugee Agency: Geneva, Switzerland, 2021. Available online: https: / / www.unhcr.org/ending-statelessness (accessed on 15 September 2020).

26. The UN Refugee Agency. In Search of Solutions: Addressing Statelessness in the Middle East and North Africa; The UN Refugee Agency: Geneva, Switzerland, 2016. Available online: https://www.refworld.org/pdfid/5829c32a4.pdf (accessed on 15 September 2020).

27. Al-Kharabsheh, L.; Al-Bazz, S.; Koc, M.; Garcia, J.; Lane, G.; Engler-Stringer, R.; White, J.; Vatanparast, H. Household food insecurity and associated socio-economic factors among recent Syrian refugees in two Canadian cities. Bord. Crossing 2020, 10, 203-214. [CrossRef]

28. The UN Refugee Agency. UN and Partners Launch Plan to Support Syrian Refugees and Countries Hosting Them as Number of Syrian Refugee New-Borns Reaches One Million Mark; The UN Refugee Agency: Geneva, Switzerland, 2018. Available online: https: / /www.unhcr.org/news/press/2018/12/5c0f80eb4/un-partners-launch-plan-support-syrian-refugees-countrieshosting-number.html (accessed on 15 September 2020).

29. Women in Informal Employment: Globalizing and Organizing. Informal Economy. 2021. Available online: https: / / www.wiego.org/informal-economy-: \{\}:text=The\%20informal\%20economy $\% 20$ is $\% 20$ the, wage $\% 20$ employment $\% 20 \mathrm{in} \% 20$ unprotected\%20jobs (accessed on 10 October 2020).

30. Tricco, A.C.; Lillie, E.; Zarin, W.; O’Brien, K.K.; Colquhoun, H.; Levac, D.; Moher, D.; Peters, M.D.J.; Horsley, T.; Weeks, L.; et al. PRISMA extension for scoping reviews (PRISMA-ScR): Checklist and explanation. Ann. Intern. Med. 2018, 169, 467-473. [CrossRef]

31. Clarivate Analytics, Endnote. Version X9 [Software]. 2021. Available online: https://endnote.com/ (accessed on 1 August 2021).

32. Abu Hamad, B.; Jones, N.; Samuels, F.; Gercama, I.; Presler-Marshall, E.; Plank, G. A Promise of Tomorrow: The Effects of UNHCR and UNICEF Cash Assistance on Syrian Refugees in Jordan; Overseas Development Institute: London, UK, 2017. Available online: https: / / odi.org/en/publications / a-promise-of-tomorrow-the-effects-of-unhcr-and-unicef-cash-assistance-on-syrianrefugees-in-jordan/ (accessed on 9 November 2020).

33. Alloush, M.; Taylor, J.E.; Gupta, A.; Rojas Valdes, R.I.; Gonzalez-Estrada, E. Economic life in refugee camps. World Dev. 2017, 95, 334-347. [CrossRef]

34. Alsamman, S. Managing infant and young child feeding in refugee camps in Jordan. Field Exch. 2014, 48, 85. Available online: https:/ / www.ennonline.net/fex/48/managinginfant (accessed on 2 November 2020).

35. Aste, N.; Barbieri, J.; Berizzi, A.; Colombo, E.; Del Pero, C.; Leonforte, F.; Merlo, M.; Riva, F. Innovative energy solutions for improving food preservation in humanitarian contexts: A case study from informal refugees settlements in Lebanon. Sustain. Energy Technol. Assess. 2017, 22, 177-187. [CrossRef]

36. Battistin, F. Impact Evaluation of the Lebanon Multipurpose Cash Assistance Programme; Lebanon Cash Consortium: Beirut, Lebanon, 2017; p. 52. Available online: https://resourcecentre.savethechildren.net/library/impact-evaluation-multipurpose-cashassistance-programme (accessed on 9 November 2020).

37. Betts, A.; Omata, N.; Sterck, O. The Kalobeyei settlement: A self-reliance model for refugees? J. Refug. Stud. 2020, 33, 189-223. [CrossRef]

38. Bloom, J.D.; Hardison-Moody, A.; Schulman, M. Bonding and bridging: Leveraging immigrant and refugee community assets to support healthy eating. Community Dev. 2018, 49, 211-230. [CrossRef]

39. Boston Consulting Group. Food-restricted voucher or unrestricted cash? In How to Best Support Syrian refugees in Jordan and Lebanon? World Food Programme: Rome, Italy, 2017. Available online: https://reliefweb.int/report/lebanon/food-restrictedvoucher-or-unrestricted-cash-how-best-support-syrian-refugees-jordan (accessed on 10 October 2020).

40. De Bruin, N.; Becker, P. Encampment and cash-based transfer: Concord and controversy in the World Food Programme's pilot project in Nyarugusu Refugee Camp in Tanzania. J. Immigr. Refug. Stud. 2019, 17, 492-508. [CrossRef]

41. Dehnavi, S.; Suss, V. Urban agriculture towards food security of Syrian refugees and vulnerable Lebanese host communities. Dev. Pract. 2019, 29, 635-644. [CrossRef]

42. Eggert, L.K.; Blood-Siefried, J.; Champagne, M.; Al-Jumaily, M.; Biederman, D.J. Coalition building for health: A community garden pilot project with apartment dwelling refugees. J. Community Health Nurs. 2015, 32, 141-150. [CrossRef]

43. El Harake, M.D.; Kharroubi, S.; Hamadeh, S.K.; Jomaa, L. Impact of a pilot school-based nutrition intervention on dietary knowledge, attitudes, behavior and nutritional status of Syrian refugee children in the Bekaa, Lebanon. Nutrients 2018, 10, 913. [CrossRef]

44. Fander, G.; Frega, M. Responding to nutrition gaps in Jordan in the Syrian refugee crisis: Infant and Young Child Feeding education and malnutrition treatment. Field Exch. 2014, 48, 82-84. Available online: https://www.ennonline.net/fex/48/responding (accessed on 9 November 2020).

45. Food and Agriculture Organization of the United Nations. Improving the Nutrition of Syrian Refugees and Host Communities through Garden Walls; Food and Agriculture Organization of the United Nations: Rome, Italy, 2016. Available online: https: / / reliefweb.int/report/lebanon/improving-nutrition-syrian-refugees-and-host-communities-through-garden-walls (accessed on 15 September 2020).

46. Food and Agriculture Organization of the United Nations. Vegetable and Staple Food Production in Refugee Settlements in Northern and Mid-Western Uganda; Food and Agriculture Organization of the United Nations: Rome, Italy, 2018. Available online: http:/ /www.fao.org/resilience/resources/resources-detail/en/c/1046064/ (accessed on 15 September 2020). 
47. Food and Agriculture Organization of the United Nations. South Sudanese Refugees Regain Livelihoods in the Democratic Republic of the Congo; Food and Agriculture Organization of the United Nations: Rome, Italy, 2020. Available online: http:/ / www.fao.org/ in-action/south-sudanese-refugees-regain-livelihoods-drc/en/ (accessed on 15 September 2020).

48. Ghattas, H.; Choufani, J.; Jamaluddine, Z.; Masterson, A.R.; Sahyoun, N.R. Linking women-led community kitchens to school food programmes: Lessons learned from the Healthy Kitchens, Healthy Children intervention in Palestinian refugees in Lebanon. Public Health Nutr. 2019, 23, 914-923. [CrossRef]

49. Gichunge, C.; Kidwaro, F. Utamu wa Afrika (the sweet taste of Africa): The vegetable garden as part of resettled African refugees' food environment. Nutr. Diet. 2014, 71, 270-275. [CrossRef]

50. Giordano, N.; Dunlop, K.; Gabay, T.; Sardiwal, D. Action Against Hunger UK's Monitoring, Evaluation and Learning Services. Evaluation Synthesis of UNHCR's Cash Based Interventions in Jordan; The UN Refugee Agency: Geneva, Switzerland, 2017. Available online: https:/ / www.unhcr.org/5a5e16607.pdf (accessed on 9 November 2020).

51. Goh, J.; Kurschner, S.; Esmail, T.; Van Arneman, J. When money speaks: Behind asylum seekers' consumption patterns. Forced Migr. Rev. 2017, 54, 91-93. Available online: https://www.fmreview.org/resettlement/goh-kurschner-esmail-vanarneman (accessed on 23 October 2020).

52. Gold, A.; Yu, N.; Buro, B.; Garden-Robinson, J. Discussion map and cooking classes: Testing the effectiveness of teaching food safety to immigrants and refugees. J. Nutr. Educ. Behav. 2014, 46, 547-553. [CrossRef] [PubMed]

53. Gunnell, S.; Christensen, N.K.; Jewkes, M.D.; LeBlanc, H.; Christofferson, D. Providing nutrition education to recently resettled refugees: Piloting a collaborative model and evaluation methods. J. Immigr. Minority Health 2015, 17, 482-488. [CrossRef]

54. Hartwig, K.A.; Mason, M. Community gardens for refugee and immigrant communities as a means of health promotion. $J$. Community Health 2016, 41, 1153-1159. [CrossRef] [PubMed]

55. Hashmi, A.; Carrara, V.I.; Nyein, P.B.; Darakamon, M.C.; Charunwatthana, P.; McGready, R. The Healthy Baby Flipbook: Piloting home-based counseling for refugee mothers to improve infant feeding and water, sanitation, and hygiene (WASH) practices. Glob. Health Action 2019, 12, 1560115. [CrossRef] [PubMed]

56. Hidrobo, M.; Hoddinott, J.; Peterman, A.; Margolies, A.; Moreira, V. Cash, food, or vouchers? Evidence from a randomized experiment in northern Ecuador. J. Dev. Econ. 2014, 107, 144-156. [CrossRef]

57. Hoddinott, J.; Dorosh, P.; Filipski, M.; Rosenbach, G.; Tiburcio, E. Food transfers, electronic food vouchers and child nutritional status among Rohingya children living in Bangladesh. PLoS ONE 2020, 15, e0230457. [CrossRef] [PubMed]

58. Ibrahim, N.; Honein-AbouHaidar, G.; Jomaa, L. Perceived impact of community kitchens on the food security of Syrian refugees and kitchen workers in Lebanon: Qualitative evidence in a displacement context. PLoS ONE 2019, 14, e0210814. [CrossRef]

59. Inglis, K.; Vargas, J. Experiences of the e-Food card programme in the Turkish refugee camps. Field Exch. 2014, 48, 145-147.

60. Karama Organization. Rooftop Gardens for Refugee Women in Deheishe Refugee Camp; Karama Organization: The West Bank, Palestine, 2015. Available online: http:/ / www.karama.org/eng/rooftop_farms.html-: \{\}:text=Rooftop\%20Gardens\%20for\%20 Refugee \%20Women\%20in\%20Deheishe\%20Refugee \%20Camp\&text=The \%20gardens \%20enable \%20the \%20women,reduces\% 20their\%20levels\%20of\%20stress (accessed on 23 October 2020).

61. Mannion, C.A.; Raffin-Bouchal, S.; Henshaw, C.J. Navigating a strange and complex environment: Experiences of Sudanese refugee women using a new nutrition resource. Int. J. Women's Health 2014, 6, 411-422. [CrossRef]

62. McElrone, M.; Colby, S.; Fouts, H.N.; Spence, M.; Kavanagh, K.; Franzen-Castle, L.; Olfert, M.F.; Kattelmann, K.K.; White, A.A. Feasibility and acceptability of implementing a culturally adapted cooking curriculum for Burundian and Congolese refugee families. Ecol. Food Nutr. 2020, 59, 598-614. [CrossRef]

63. Millican, J.; Perkins, C.; Adam-Bradford, A. Gardening in displacement: The benefits of cultivating in crisis. J. Refug. Stud. 2019, 32, 351-371. [CrossRef]

64. Mochizuki, Y. The livelihood strategies of South Sudanese in a refugee settlement in Uganda. Localization Humanit. Assist. Framew. East Afr. Pastor. 2017, 53, 131-139.

65. Ngwenyi, E. Addressing acute malnutrition in Cameroon during an emergency: Results and benefits of an integrated prevention programme. Field Exch. 2019, 60, 96-100.

66. Oka, R. Unlikely cities in the desert: The informal economy as causal agent for permanent "urban" sustainability in Kakuma Refugee Camp, Kenya. Urban Anthropol. 2011, 40, 223-262.

67. Pavanello, S. Multi-Purpose Cash and Sectoral Outcomes: Greece Case Study; The UN Refugee Agency: Geneva, Switzerland, 2018. Available online: https:/ / www.unhcr.org/protection/operations/5b2cfa1f7/multi-purpose-cash-sectoral-outcomes-case-studygreece.html (accessed on 25 October 2020).

68. Qleibo, E.; Nada, E.A.; Mushtaha, W.; Campbell, J. Cash voucher programme and rabbit raising intervention in Gaza. Field Exch.-Emerg. Nutr. Netw. ENN 2013, 46, 10-12. Available online: https://www.ennonline.net/fex/46/cash (accessed on 8 November 2020).

69. Sebuliba, H.; El-Zubi, F. Meeting Syrian refugee children and women nutritional needs in Jordan. Field Exch. 2014, 48, 74-75. Available online: https:/ / www.ennonline.net/fex/48/meetingsyrian (accessed on 8 November 2020).

70. Smock, L.; Martelon, M.; Metallinos-Katsaras, E.; Nguyen, T.; Cochran, J.; Geltman, P.L. Recovery from malnutrition among refugee children following participation in the special supplemental nutrition for Women, Infants, and Children (WIC) program in Massachusetts, 1998-2010. J. Public Health Manag. Pract. 2020, 26, 71-79. [CrossRef] 
71. Stuetz, W.; Carrara, V.I.; McGready, R.; Lee, S.J.; Sriprawat, K.; Po, B.; Hanboonkunupakarn, B.; Grune, T.; Biesalski, H.K.; Nosten, F.H. Impact of food rations and supplements on micronutrient status by trimester of pregnancy: Cross-sectional studies in the Maela refugee camp in Thailand. Nutrients 2016, 8, 66. [CrossRef]

72. SüSS, V. Role of urban agriculture to increase food security and economic resilience of refugees and vulnerable host communities. In The Case of Syrian Refugees in Bourj Hammoud, Lebanon; Cologne University of Applied Science: Köln, Germany, 2018.

73. Tomkins, M.; Yousef, S.; Adam-Bradford, A.; Perkins, C.; Grosrenaud, E.; McTough, M.; Viljoen, A. Cultivating refuge: The role of urban agriculture amongst refugees and forced migrants in the Kurdistan region of Iraq. Int. J. Des. Nat. Ecodynamics 2019, 14, 103-118. Available online: https:/ / reliefweb.int/report/iraq/cultivating-refuge-role-urban-agriculture-amongst-refugeesand-forced-migrants-kurdistan (accessed on 8 November 2020). [CrossRef]

74. Trapp, M. What's on the table: Nutrition programming for refugees in the United States. NAPA Bull. 2010, 34, 161-175. [CrossRef]

75. Volpato, G.; Rossi, D.; Dentoni, D. A reward for patience and suffering: Ethnomycology and commodification of desert truffles among Sahrawi refugees and nomads of Western Sahara. Econ. Bot. 2013, 67, 147-160. [CrossRef]

76. Volpato, G. Exile, Camps, and Camels: Recovery and Adaptation of Subsistence Practices and Ethnobiological Knowledge among Sahrawi Refugees; Wageningen University: Wageningen, The Netherlands, 2014. Available online: https:/ / edepot.wur.nl/317117 (accessed on 9 November 2020).

77. Wilson, A.; Szwed, N.; Renzaho, A. Developing nutrition guidelines for recycled food to improve food security among homeless, asylum seekers, and refugees in Victoria, Australia. J. Hunger. Environ. Nutr. 2012, 7, 239-252. [CrossRef]

78. World Food Programme. Mozambique PRRO 200355 Assistance to Vulnerable Groups and Disaster Affected Populations: An Operation Evaluation; World Food Programme: Rome, Italy, 2014; Available online: https:/ / www.wfp.org/publications/mozambique-prro200355-assistance-vulnerable-groups-and-disaster-affected-populations-operat (accessed on 18 September 2020).

79. World Food Programme. Iran PRRO 200310 Food Assistance and Education Incentive for Afghan and Iraqi Refugees (2013-2015): A Mid-Term Operation Evaluation; World Food Programme: Rome, Italy, 2015. Available online: https://www.wfp. org/publications/iran-prro-200310-food-assistance-and-education-incentive-afghan-and-iraqi-refugees-2013-2015\#: \{\}: text $=1 \% 20$ March $\% 202015$ - Iran $\% 20$ PRRO $\% 20200310 \% 20$ Food $\% 20$ Assistance $\% 20$ and $\% 20$ education $\% 20$ incentive $\% 20$ for $\% 20$ Afghan,A\%20mid\%2Dterm\%20Operation\%20Evaluation\&text=The \%20evaluation $\% 20$ assessed $\% 20$ the $\% 20$ following,meals $\%$ 3B\%20and\%20Food\%20For\%20Training (accessed on 15 September 2020).

80. World Food Programme. Ethiopia PRRO 200700 (2015-2018) Food Assistance for Eritrean, South Sudanese, Sudanese and Somali refugees: An Operation Evaluation; World Food Programme: Rome, Italy, 2016. Available online: https://www.wfp.org/ publications / ethiopia-prro-200700-2015-2018-food-assistance-eritrean-south-sudanese-sudanese-and-somali-r (accessed on 15 September 2020).

81. World Food Programme. Liberia PRRO 200550 Food Assistance for Refugees and Vulnerable Host Populations: An Operation Evaluation; World Food Programme: Rome, Italy, 2016. Available online: https:/ /www.wfp.org/publications/liberia-prro-200550-foodassistance-refugees-and-vulnerable-host-populations-operation-evalu (accessed on 18 September 2020).

82. World Food Programme. Rwanda PRRO 200744 Food and Nutrition Assistance to Refugees and Returnees: A Mid-Term Operation Evaluation; World Food Programme: Rome, Italy, 2016. Available online: https:/ / www.wfp.org/publications/rwanda-prro-2007 44-food-and-nutrition-assistance-refugees-and-returnees-mid-term-operation-e (accessed on 16 September 2020).

83. World Food Programme. Kenya, General Food Distribution Cash Modality Scale up for the Refugees and Host Community in Kakuma and Dadaab Camp: An Evaluation; World Food Programme: Rome, Italy, 2018. Available online: https://www.wfp.org/publications/ kenya-general-food-distribution-cash-modality-evaluation-terms-reference\#: \{\}:text=1\%20December\%202018-,Kenya\%2C \% 20General\%20Food\%20Distribution\%20Cash\%20Modality \%20scale $\% 20$ u $\% 20$ for $\% 20$ the, and $\% 20$ Dadaab $\% 20$ Camp $\% 3 \mathrm{~A} \%$ 20an \%20 evaluation\&text=Key\%20evaluation\%20findings\%20included\%3A,USD1\%20to\%20beneficiaries \%20was\%20USD1 (accessed on 19 September 2020).

84. World Food Programme. Algeria PRRO 200301: Evaluation of the Nutrition Components; World Food Programme: Rome, Italy, 2018. Available online: https://www.wfp.org/publications/algeria-prro-200301-evaluation-nutrition-components\#: \{\}: text=This\%20decentralized\%20evaluation\%20was\%20commissioned,October\%202017\%20and\%20July\%202018 (accessed on 27 December 2021).

85. World Food Programme. Cameroon: An Evaluation of WFP's Portfolio (2012-2017); World Food Programme: Rome, Italy, 2018. Available online: https://www.wfp.org/publications/cameroon-evaluation-wfps-portfolio-2012-2017 (accessed on 19 September 2020).

86. WTsadik, M. The experience of multi-storey gardens in Ethiopian refugee camps. Field Exch. 2011, 40, 35-37.

87. Food and Agriculture Organization of the United Nations. About FAO; Food and Agriculture Organization of the United Nations: Rome, Italy, 2022. Available online: https:/ /www.fao.org/about/en/ (accessed on 4 July 2021).

88. The UN Refugee Agency, World Food Programme. Memorandum of Understanding between the Office of the United Nations High Commissioner for Refugees (UNHCR) and the World Food Programme (WFP); The UN Refugee Agency: Geneva, Switzerland, 2011. Available online: https://www.unhcr.org/publications/operations/53465c929/memorandum-understanding-unhcr-wfpjanuary-2011.html (accessed on 27 December 2021).

89. The UN Refugee Agency, World Food Programme. Joint Strategy: Enhancing Self-Reliance in Food Security and Nutrition in Protracted Refugee Situations; The UN Refugee Agency: Geneva, Switzerland, 2020. Available online: https://reliefweb.int/report/world/ wfp-unhcr-joint-strategy-enhancing-self-reliance-food-security-and-nutrition-protracted (accessed on 27 December 2021). 
90. Moore, E.A.; Munsell, J.F.; Hammett, A.L.T.; Moore, K.M. Agroforestry preferences in refugee hosting communities in Cameroon. Agrofor. Syst. 2014, 88, 735-752. [CrossRef]

91. Lane, G.; Nisbet, C.; Vatanparast, H. Food insecurity and nutritional risk among Canadian newcomer children in Saskatchewan Nutrients 2019, 11, 1744. [CrossRef] [PubMed]

92. Government of Canada. Household Food Insecurity in Canada in 2007-2008: Key Statistics and Graphics. Canadian Community Health Survey; Government of Canada: Ottawa, ON, Canada, 2012. Available online: https://www.canada.ca/en/healthcanada/services/food-nutrition/food-nutrition-surveillance/health-nutrition-surveys/canadian-community-health-surveycchs/household-food-insecurity-canada-overview / household-food-insecurity-canada-2007-2008-key-statistics-graphicsfood-nutrition-surveillance-health-canada.html (accessed on 27 December 2021).

93. Canadian Council for Refugees. Refugees Receive Limited, If Any, Social Assistance from Government Authorities. Available online: https:/ / ccrweb.ca/en/refugees-social-assistance (accessed on 27 December 2021).

94. The UN Refugee Agency. Gender-Based Violence; The UN Refugee Agency: Geneva, Switzerland, 2021. Available online: https: / / www.unhcr.org/gender-based-violence.html (accessed on 27 December 2021).

95. World Food Programme. Consolidated Approach to Reporting Indicators of Food Security (CARI); United Nations World Food Programme, Food Security Analysis (VAM): Rome, Italy, 2015.

96. Government of Canada. The Household Food Security Survey Module (HFSSM). 2012. Available online: https://www.canada. $\mathrm{ca} /$ en/health-canada/services/food-nutrition/food-nutrition-surveillance/health-nutrition-surveys/canadian-communityhealth-survey-cchs/household-food-insecurity-canada-overview/household-food-security-survey-module-hfssm-healthnutrition-surveys-health-canada.html (accessed on 27 December 2021).

97. The UN Refugee Agency, World Food Programme. Joint Principles for Targeting Assistance to Meet Food and other Basic Needs to Persons of Concern; The UN Refugee Agency, World Food Programme: Rome, Italy, 2017; Available online: https:/ / www.unhcr. org/protection/health/5ad9e6407/unhcr-wfp-joint-principles-targeting-assistance-meet-food-other-basic-needs.html (accessed on 27 December 2021).

98. Food and Agriculture Organization of the United Nations. Policy Guidance Series: Strengthening Sector Policies for better Food Security and Nutriiton Results; Food and Agriculture Organization of the United Nations: Rome, Italy, 2017. Available online: https://www.fao.org/publications/policy-guidance-series/en/ (accessed on 27 December 2021). 